\title{
Methodologies used in cost-effectiveness models for evaluating treatments in major depressive disorder: a systematic review
}

Evelina A Zimovetz ${ }^{1 *}$, Sorrel E Wolowacz ${ }^{1 \dagger}$, Peter M Classi ${ }^{2+}$ and Julie Birt ${ }^{2 \dagger}$

\begin{abstract}
Background: Decision makers in many jurisdictions use cost-effectiveness estimates as an aid for selecting interventions with an appropriate balance between health benefits and costs. This systematic literature review aims to provide an overview of published cost-effectiveness models in major depressive disorder (MDD) with a focus on the methods employed. Key components of the identified models are discussed and any challenges in developing models are highlighted.

Methods: A systematic literature search was performed to identify all primary model-based economic evaluations of MDD interventions indexed in MEDLINE, the Cochrane Library, EMBASE, EconLit, and PsycINFO between January 2000 and May 2010.
\end{abstract}

Results: A total of 37 studies were included in the review. These studies predominantly evaluated antidepressant medications. The analyses were performed across a broad set of countries. The majority of models were decisiontrees; eight were Markov models. Most models had a time horizon of less than 1 year. The majority of analyses took a payer perspective. Clinical input data were obtained from pooled placebo-controlled comparative trials, single head-to-head trials, or meta-analyses. The majority of studies (24 of 37) used treatment success or symptomfree days as main outcomes, 14 studies incorporated health state utilities, and 2 used disability-adjusted life-years. A few models (14 of 37) incorporated probabilities and costs associated with suicide and/or suicide attempts. Two models examined the cost-effectiveness of second-line treatment in patients who had failed to respond to initial therapy. Resource use data used in the models were obtained mostly from expert opinion. All studies, with the exception of one, explored parameter uncertainty.

Conclusions: The review identified several model input data gaps, including utility values in partial responders, efficacy of second-line treatments, and resource utilisation estimates obtained from relevant, high-quality studies. It highlighted the differences in outcome measures among the trials of MDD interventions, which can lead to difficulty in performing indirect comparisons, and the inconsistencies in definitions of health states used in the clinical trials and those used in utility studies. Clinical outcomes contributed to the uncertainty in cost-effectiveness estimates to a greater degree than costs or utility weights.

Keywords: Systematic review, cost-effectiveness analysis, decision analysis, major depressive disorder

\footnotetext{
* Correspondence: ezimovetz@rti.org

+ Contributed equally

'RTI Health Solutions, The Pavilion, Towers Business Park, Wilmslow Road,

Didsbury, Manchester, M20 2LS, UK

Full list of author information is available at the end of the article
}

\section{Ciomed Central}

(C) 2012 Zimovetz et al; licensee BioMed Central Ltd. This is an Open Access article distributed under the terms of the Creative Commons Attribution License (http://creativecommons.org/licenses/by/2.0), which permits unrestricted use, distribution, and reproduction in any medium, provided the original work is properly cited. 


\section{Introduction}

Major depressive disorder (MDD) is a highly prevalent condition estimated to affect $2.3 \%$ of the global population [1]. MDD is associated with decreased patient wellbeing [2], significant burden on health care costs, and productivity losses [3]. It is projected that by the year 2020, depression will rank second in disease burden measured by disability-adjusted life-years [4]. The most common and generally accepted treatment options for patients with MDD include pharmacotherapy, psychotherapy, and pharmacotherapy in combination with psychotherapy [5]. A variety of pharmacotherapies exist for treating MDD; traditionally, these fall into pharmacological classes, such as tricyclic antidepressants (TCAs), tetracyclic antidepressants (non-selective serotonin and norepinephrine reuptake inhibitors), selective serotonin reuptake inhibitors (SSRIs), selective norepinephrine reuptake inhibitors (NRIs), selective serotonin and norepinephrine reuptake inhibitors (SNRIs), monoamine oxidase inhibitors (MAOIs) (including irreversible MAOIs and reversible inhibitors of monoamine oxidase A [RIMAs]), agonists of the melatonin receptor (MT agonists), and other antidepressants [6]. In addition, a wide range of psychotherapeutic options are available, including behavioural therapy, interpersonal therapy, cognitive behavioural therapy (CBT), and the cognitive behavioural analysis system of psychotherapy [6].

With limitations on health care spending, it is important to allocate resources to interventions that are seen to maximise cost-effectiveness. Evaluating the cost-effectiveness of alternative treatment options in MDD can shape policies concerning formulary coverage and reimbursement. A significant number of models evaluating the cost-effectiveness of alternative MDD strategies have been developed. Some of these were examined by Barrett and colleagues [7] in their systematic review of published economic evaluations of interventions for depression. However, no systematic review of the decision-analytic models in MDD has been published recently.

The objectives of this systematic review were to identify published decision-analytic models evaluating the cost-effectiveness of pharmacological treatments in MDD; to examine the variation and frequency of methods employed, highlighting advantages and disadvantages in these methodologies; and to identify specific areas in the MDD cost-effectiveness literature that merited further research to allow improvement in the quality of the economic evaluations.

\section{Methods}

A systematic literature search was performed to identify relevant articles with abstracts indexed in MEDLINE, the Cochrane Library, EMBASE, EconLit, and
PsycINFO. A search strategy was developed for each electronic database using a combination of Medical Subject Heading $(\mathrm{MeSH})$ and free-text terms, grouped into the following categories: disease, interventions, economics, and study type. MeSH terms used were 'Depressive Disorder, Major', 'Drug Therapy', 'Antidepressive Agents', 'Costs and Cost Analysis', 'Cost-Benefit Analysis', 'Economics, Hospital', 'Economics, Medical', 'Economics, Nursing', 'Economics, Pharmaceutical', 'Fees and Charges', 'Health Resources/utilization'. The search was limited to articles published in the English language from January 2000 to May 2010. The search strategies were developed by an information specialist with input from authors; full details can be made available on request. Reference lists of identified review articles were checked for relevant studies.

Predefined inclusion criteria were used to determine the selection of the studies. The studies of interest included model-based economic evaluations of pharmacological interventions in MDD (e.g., Markov models, decision-tree models, and models based on mathematical equations). Excluded studies were reviews, editorials, resource use and cost studies, and economic evaluations alongside a clinical trial or those evaluating non-pharmacological interventions only. Study inclusion was performed by the authors and disagreements were resolved by consensus. Data extraction included author and year, country of analysis, analysis type and model structure, analysis time horizon and perspective, treatment comparators, primary outcomes, definitions of effectiveness, sources of primary clinical data, sources of utility data, treatment of uncertainty, and main findings. For each eligible study, data of interest were extracted by one researcher. To ensure quality and accuracy of the data, a second researcher verified the extracted data with their original sources.

\section{Results}

\section{Search Results and Study Characteristics}

The search retrieved 1320 citations, 34 of which met the study inclusion criteria, and a further 3 were identified through screening of the reference lists (Figure 1). The characteristics of the included economic models are presented in Table 1[8-45]. Of the 37 included studies, 23 were cost-effectiveness analyses and 14 were cost-utility analyses. The majority of the models (28 of 37) had a decision-tree structure, eight described themselves as Markov models [9-11,16,26,32,33,37], and one [31] did not explicitly report the model structure.

The majority of studies (22 of 37) adopted a 6-month time horizon; most were conducted from the health care payer perspective (19 of 37) and were in adults with MDD receiving first-line therapy (32 of 37).

Of the included studies, 29 examined pharmacological interventions only, four studies evaluated non- 


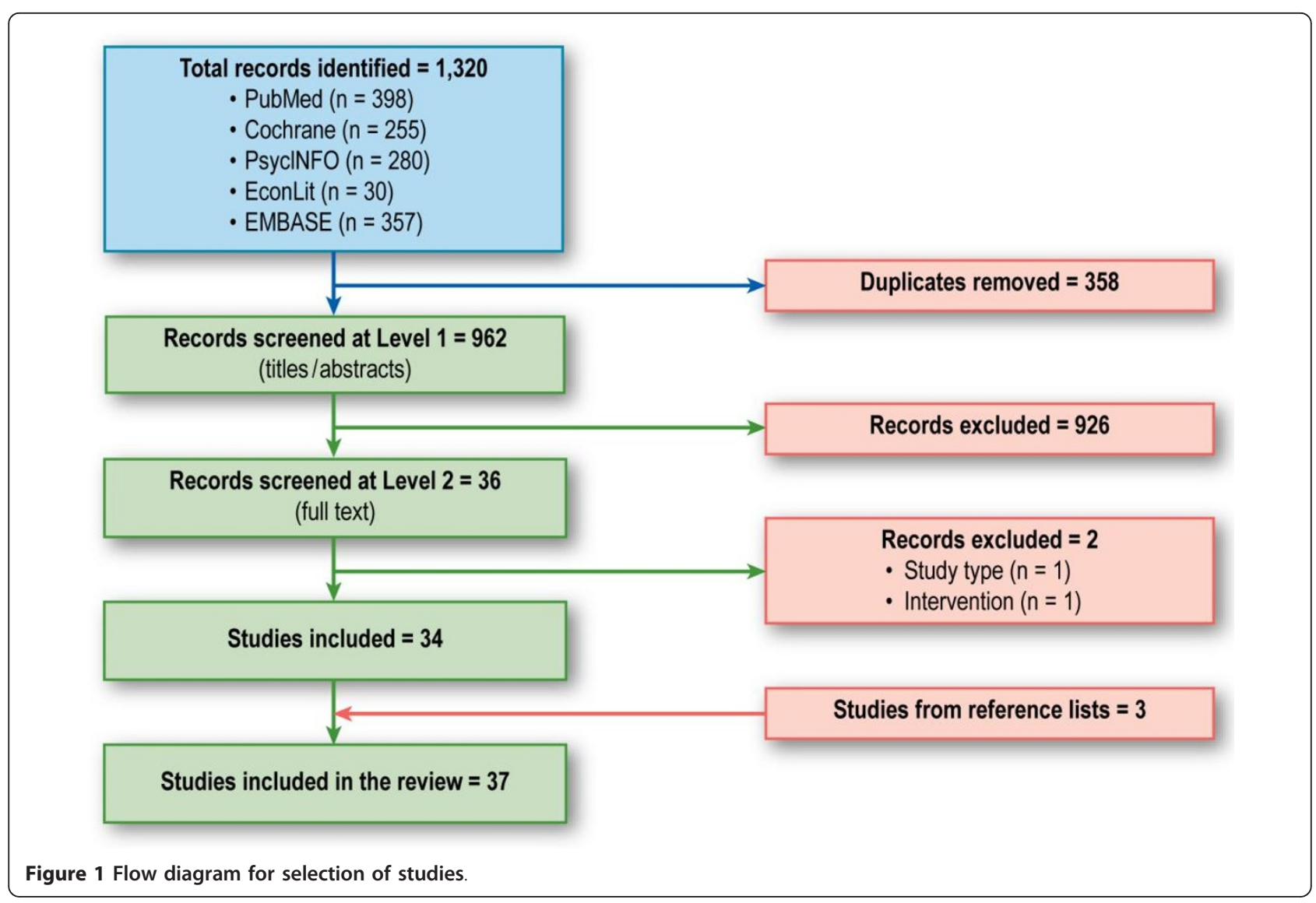

pharmacological interventions versus pharmacological therapies $[10,16,22,42]$, two studies included comparisons of pharmacological treatments versus combination therapies (i.e., pharmacotherapy and behavioural therapy) $[35,36]$, one study evaluated the prolongation of pharmacological treatment versus no prolongation following response to initial therapy [32], and one study evaluated pharmacogenetic testing for antidepressant response [33].

The included studies represented 23 countries: Australia [22,42], Austria [23,24], Belgium [17], Brazil [30], Canada [41], Denmark [38], Finland [19], France [13,16], Germany [14,18,26], Italy [14,18], Japan [35], Norway [20], Poland [14,18], Scotland [11], Singapore [45], Spain $[14,18]$, Sweden $[14,18,29,37]$, Switzerland [14,18], Thailand [25], the Netherlands [14,18,32], United Kingdom (UK) $[12,14,18,21,27,28,36,43,44]$, United States (US) $[8-10,14,15,18,31,33,39,40]$, and Venezuela $[14,18]$.

\section{Modelling Approaches}

Of the identified models, 28 were described as decisiontree models. Of these, nine [17,19,20,23,25,29,38,43,45] had a similar design based on the model structure reported by Francois [19] (Figure 2). The decision-tree structure used by these models consisted of two paths.
Patients with MDD entered the model in the first path representing primary care. Patients with inadequate response in primary care could titrate to a higher dose or switch treatment. Patients with insufficient response after titration and/or switching were referred to secondary care, represented by the second path. In the secondary care path, patients could have their dose titrated, have their treatment switched, receive adjunctive therapy with another agent, or be hospitalised. The model design incorporated the rates of suicide and suicide attempt. Most of the models used the rates of suicide and attempted suicide reported by Khan [46], with two models $[19,20]$ using alternative sources. Three of the studies $[25,43,45]$ that adapted the model structure reported by Francois [19] performed country-specific modifications to better reflect local clinical practice.

Of the decision-tree models identified, six $[14,15,18,21,27,28]$ were based on the structure presented by Casciano [15] (Figure 3). The events modelled following treatment failure due to lack of efficacy included titration to maximum dosage, within-class adjunctive therapy, between-class adjunctive therapy, and treatment switch. Chance nodes for these events were evaluated through consultation with clinical experts. The analysis by Doyle and colleagues [18] 
Table 1 Summary of methods and conclusions of included studies

\begin{tabular}{|c|c|c|c|c|c|c|c|c|}
\hline $\begin{array}{l}\text { Author, Year, } \\
\text { Country }\end{array}$ & Compara-tors & $\begin{array}{l}\text { Analysis } \\
\text { Type, } \\
\text { Model } \\
\text { Structure }\end{array}$ & $\begin{array}{l}\text { Time } \\
\text { Horizon, } \\
\text { Perspective }\end{array}$ & $\begin{array}{l}\text { Primary } \\
\text { Out-come }\end{array}$ & $\begin{array}{l}\text { Definition of } \\
\text { Effective-ness }\end{array}$ & $\begin{array}{l}\text { Source of } \\
\text { Primary } \\
\text { Clinical Data }\end{array}$ & $\begin{array}{l}\text { Sensitivity } \\
\text { Analyses }\end{array}$ & Main Finding \\
\hline $\begin{array}{l}\text { Armstrong et } \\
\text { al., } 2007 \text { [8] } \\
\text { US }\end{array}$ & $\begin{array}{l}\text { Escitalopram } \\
\text { vs. } \\
\text { sertraline }\end{array}$ & $\begin{array}{l}\text { CUA; } \\
\text { decision- } \\
\text { tree }\end{array}$ & $\begin{array}{l}6 \text { months, } \\
\text { payer } \\
\text { perspective }\end{array}$ & QALYS & $\begin{array}{l}\text { Response: } \geq 50 \% \\
\text { improve-ment in } \\
\text { MADRS }\end{array}$ & $\begin{array}{l}\text { 8-week head- } \\
\text { to-head trial }\end{array}$ & $\begin{array}{l}\text { Univariate, } \\
\text { probabilistic }\end{array}$ & $\begin{array}{l}\text { Escitalopram } \\
\text { dominated }^{\mathrm{a}} \text { sertraline }\end{array}$ \\
\hline $\begin{array}{l}\text { Armstrong et } \\
\text { al., } 2008 \text { [9] } \\
\text { US }\end{array}$ & $\begin{array}{l}\text { Escitalopram } \\
\text { vs. } \\
\text { duloxetine }\end{array}$ & $\begin{array}{l}\text { CUA; } \\
\text { Markov } \\
\text { structure; } \\
\text { 1-week } \\
\text { cycle }\end{array}$ & $\begin{array}{l}1 \text { year, } \\
\text { payer } \\
\text { perspective }\end{array}$ & QALWS & $\begin{array}{l}\text { Remission: MADRS } \leq \\
12 \text { or HAMD-17 } \leq 7\end{array}$ & $\begin{array}{l}\text { Pooled } \\
\text { analysis of } 10 \\
\text { RCTs }\end{array}$ & $\begin{array}{l}\text { Univariate, } \\
\text { probabilistic }\end{array}$ & $\begin{array}{l}\text { Escitalopram } \\
\text { dominated }^{\mathrm{a}} \text { duloxetine }\end{array}$ \\
\hline $\begin{array}{l}\text { Aziz et al., } \\
2005[10] \\
\text { US }\end{array}$ & $\begin{array}{l}\text { MPT vs. } \\
\text { MECT }^{b}\end{array}$ & $\begin{array}{l}\text { CUA; } \\
\text { Markov } \\
\text { structure; } \\
\text { 6-month } \\
\text { cycle }\end{array}$ & $\begin{array}{l}\text { Lifetime, } \\
\text { payer and } \\
\text { societal } \\
\text { perspective }\end{array}$ & QALYS & $\begin{array}{l}\text { Remission: not } \\
\text { explicitly defined }\end{array}$ & $\begin{array}{l}\text { Published } \\
\text { literature }\end{array}$ & Univariate & $\begin{array}{l}\text { MECT may be more } \\
\text { cost-effective vs. MPT }\end{array}$ \\
\hline \multirow[t]{2}{*}{$\begin{array}{l}\text { Benedict et al., } \\
2010[11] \\
\text { Scotland }\end{array}$} & $\begin{array}{l}\text { Primary care: } \\
\text { duloxetine vS. } \\
\text { SSRIs, } \\
\text { venlafaxine ER, } \\
\text { mirtazapine }\end{array}$ & $\begin{array}{l}\text { CUA; } \\
\text { Markov } \\
\text { structure; } \\
\text { 8-week } \\
\text { cycle }\end{array}$ & $\begin{array}{l}48 \text { weeks, } \\
\text { payer } \\
\text { perspective }\end{array}$ & QALYS & $\begin{array}{l}\text { Remission and } \\
\text { response: HAMD-17 } \\
\text { scale, scores not } \\
\text { reported }\end{array}$ & $\begin{array}{l}\text { Pooled } \\
\text { analysis of } 8 \\
\text { RCTs, a meta- } \\
\text { analysis }\end{array}$ & $\begin{array}{l}\text { Univariate, } \\
\text { probabilistic }\end{array}$ & $\begin{array}{l}\text { For the commonly } \\
\text { accepted WTP } \\
\text { thresholds, duloxetine } \\
\text { was the preferred } \\
\text { option }\end{array}$ \\
\hline & $\begin{array}{l}\text { Secondary } \\
\text { care: } \\
\text { duloxetine vs. } \\
\text { venlafaxine ER, } \\
\text { mirtazapine }\end{array}$ & & & & & $\begin{array}{l}\text { Pooled } \\
\text { analysis of } 2 \\
\text { head-to-head } \\
\text { trials, a meta- } \\
\text { analysis }^{d}\end{array}$ & & $\begin{array}{l}\text { Duloxetine dominated }^{a} \\
\text { venlafaxine ER and } \\
\text { mirtazapine }\end{array}$ \\
\hline \multirow[t]{2}{*}{$\begin{array}{l}\text { Borghi and } \\
\text { Guest, } 2000 \\
{[12]} \\
\text { UK }\end{array}$} & $\begin{array}{l}\text { Mirtazapine vs. } \\
\text { amitriptyline }\end{array}$ & $\begin{array}{l}\text { CEA; } \\
\text { decision- } \\
\text { tree }\end{array}$ & $\begin{array}{l}7 \text { months, } \\
\text { payer } \\
\text { perspective, } \\
\text { societal } \\
\text { perspective }\end{array}$ & $\begin{array}{l}\text { Treatment } \\
\text { success }\end{array}$ & $\begin{array}{l}\text { Remission: } \\
\text { HAMD-17 } \leq 7\end{array}$ & $\begin{array}{l}\text { Meta-analysis } \\
\text { of } 4 \text { RCTs of } \\
\text { 7-month } \\
\text { duration }\end{array}$ & Univariate & $\begin{array}{l}\text { Mirtazapine was cost- } \\
\text { effective vs. amitriptyline } \\
\text { and fluoxetine }\end{array}$ \\
\hline & $\begin{array}{l}\text { Mirtazapine vs. } \\
\text { fluoxetine }\end{array}$ & & $\begin{array}{l}6 \text { months, } \\
\text { payer } \\
\text { perspective, } \\
\text { societal } \\
\text { perspective }\end{array}$ & $\begin{array}{l}\text { Treatment } \\
\text { success }\end{array}$ & $\begin{array}{l}\text { Response: } \geq 50 \% \\
\text { improve-ment in } 17 \text { - } \\
\text { HAMD }\end{array}$ & $\begin{array}{l}\text { 6-week head- } \\
\text { to-head trial }\end{array}$ & & \\
\hline $\begin{array}{l}\text { Brown et al., } \\
2000[13] \\
\text { France }\end{array}$ & $\begin{array}{l}\text { Mirtazapine vs. } \\
\text { fluoxetine }\end{array}$ & $\begin{array}{l}\text { CEA; } \\
\text { decision- } \\
\text { tree }\end{array}$ & $\begin{array}{l}6 \text { months, } \\
\text { societal } \\
\text { perspective }\end{array}$ & $\begin{array}{l}\text { Treatment } \\
\text { success }\end{array}$ & $\begin{array}{l}\text { Response: } \geq 50 \% \\
\text { improve-ment in } 17 \text { - } \\
\text { HAMD, HAMD-21, or } \\
\text { a score of } 1 \text { or } 2 \text { on } \\
\text { CGl }\end{array}$ & $\begin{array}{l}\text { 6-week head- } \\
\text { to-head trial }\end{array}$ & Univariate & $\begin{array}{l}\text { Mirtazapine was cost- } \\
\text { effective vs. fluoxetine }\end{array}$ \\
\hline $\begin{array}{l}\text { Casciano et al., } \\
2001[14] \\
10 \text { countries }^{\mathrm{e}}\end{array}$ & $\begin{array}{l}\text { Venlafaxine ER } \\
\text { vs. } \\
\text { SSRIs, } \\
\text { TCAs }\end{array}$ & $\begin{array}{l}\text { CEA; } \\
\text { decision- } \\
\text { tree }\end{array}$ & $\begin{array}{l}6 \text { months, } \\
\text { payer } \\
\text { perspective }\end{array}$ & $\begin{array}{l}\text { Treatment } \\
\text { success, } \\
\text { SFDs }^{\dagger}\end{array}$ & $\begin{array}{l}\text { Response: } 50 \% \\
\text { improve-ment in } \\
\text { HAMD or MADRS }\end{array}$ & Meta-analysis & $\begin{array}{l}\text { Univariate, } \\
\text { probabilistic }\end{array}$ & $\begin{array}{l}\text { Venlafaxine ER } \\
\text { dominated } \text { SSRIs and }^{\mathrm{a}} \text { SOAs in } 9 \text { of the } 10 \\
\text { countries }\end{array}$ \\
\hline $\begin{array}{l}\text { Casciano et al., } \\
2000 \text { [15] } \\
\text { US }\end{array}$ & $\begin{array}{l}\text { Venlafaxine ER } \\
\text { vs. } \\
\text { SSRIs, } \\
\text { TCAs }\end{array}$ & $\begin{array}{l}\text { CEA; } \\
\text { decision- } \\
\text { tree }\end{array}$ & $\begin{array}{l}6 \text { months, } \\
\text { payer } \\
\text { perspective }\end{array}$ & $\begin{array}{l}\text { Treatment } \\
\text { success, }^{\text {SFDs }}\end{array}$ & $\begin{array}{l}\text { Response: } 50 \% \\
\text { improve-ment in } \\
\text { HAMD or MADRS }\end{array}$ & Meta-analysis & $\begin{array}{l}\text { Univariate, } \\
\text { probabilistic }\end{array}$ & $\begin{array}{l}\text { Venlafaxine ER } \\
\text { dominated }{ }^{\mathrm{a}} \text { SSRIs and } \\
\text { TCAs }\end{array}$ \\
\hline $\begin{array}{l}\text { Dardennes et } \\
\text { al., } 2000[16] \\
\text { France }\end{array}$ & $\begin{array}{l}\text { Preventative } \\
\text { strategy }^{9} \text { vs. } \\
\text { episodic } \\
\text { strategy }^{h}\end{array}$ & $\begin{array}{l}\text { CUA; } \\
\text { Markov } \\
\text { structure; } \\
\text { 8-week } \\
\text { cycle } \\
\end{array}$ & $\begin{array}{l}12 \text { months, } \\
\text { payer } \\
\text { perspective }\end{array}$ & QALYS & $\begin{array}{l}\text { Remission: HDRS-21 } \\
<8\end{array}$ & $\begin{array}{l}\text { 12-month } \\
\text { double-blind } \\
\text { trial }\end{array}$ & Univariate & $\begin{array}{l}\text { Cost of maintenance } \\
\text { therapy was partially } \\
\text { offset by the gain from } \\
\text { recurrence prevention }\end{array}$ \\
\hline $\begin{array}{l}\text { Demyttenaere } \\
\text { et al., } 2005 \\
{[17]} \\
\text { Belgium }\end{array}$ & $\begin{array}{l}\text { Escitalopram } \\
\text { vs. } \\
\text { citalopram, } \\
\text { venlafaxine }\end{array}$ & $\begin{array}{l}\text { CEA; } \\
\text { decision- } \\
\text { tree }\end{array}$ & $\begin{array}{l}6 \text { months, } \\
\text { payer and } \\
\text { societal } \\
\text { perspec- } \\
\text { tives }\end{array}$ & $\begin{array}{l}\text { Treatment } \\
\text { success }\end{array}$ & $\begin{array}{l}\text { Remission: MADRS } \leq \\
12\end{array}$ & $\begin{array}{l}\text { Meta-analysis } \\
\text { of three 8- } \\
\text { week RCTs }\end{array}$ & $\begin{array}{l}\text { Univariate, } \\
\text { probabilistic }\end{array}$ & $\begin{array}{l}\text { Escitalopram } \\
\text { dominated }{ }^{a} \text { citalopram } \\
\text { and was cost-effective } \\
\text { vs. venlafaxine }\end{array}$ \\
\hline
\end{tabular}


Table 1 Summary of methods and conclusions of included studies (Continued)

\begin{tabular}{|c|c|c|c|c|c|c|c|c|}
\hline $\begin{array}{l}\text { Doyle et al., } \\
2001 \text { [18] }^{\mathrm{e}} \text { countries }^{\mathrm{e}}\end{array}$ & $\begin{array}{l}\text { Venlafaxine vs. } \\
\text { SSRIs, } \\
\text { TCAs }\end{array}$ & $\begin{array}{l}\text { CEA; } \\
\text { decision- } \\
\text { tree }\end{array}$ & $\begin{array}{l}6 \text { months, } \\
\text { payer } \\
\text { perspective }\end{array}$ & $\begin{array}{l}\text { Treatment } \\
\text { success, } \\
\text { SFDs }^{f}\end{array}$ & $\begin{array}{l}\text { Response: } 50 \% \\
\text { improve-ment in } \\
\text { HAMD or MADRS }\end{array}$ & $\begin{array}{l}2 \text { meta- } \\
\text { analyses }\end{array}$ & $\begin{array}{l}\text { Univariate, } \\
\text { probabilistic }\end{array}$ & $\begin{array}{l}\text { Venlafaxine dominated }^{a} \\
\text { SSRIs and TCAs in } 9 \text { of } \\
10 \text { countries (inpatients); } \\
8 \text { of } 10 \text { countries } \\
\text { (outpatients) }\end{array}$ \\
\hline $\begin{array}{l}\text { Francois et al., } \\
2002 \text { [19] } \\
\text { Finland }\end{array}$ & $\begin{array}{l}\text { Escitalopram } \\
\text { vs. } \\
\text { citalopram, } \\
\text { fluoxetine, } \\
\text { venlafaxine }\end{array}$ & $\begin{array}{l}\text { CUA; } \\
\text { decision- } \\
\text { tree }\end{array}$ & $\begin{array}{l}6 \text { months, } \\
\text { societal } \\
\text { perspective }\end{array}$ & $\begin{array}{l}\text { Treatment } \\
\text { success, } \\
\text { QALYs }\end{array}$ & $\begin{array}{l}\text { Remission: MADRS } \leq \\
12\end{array}$ & $\begin{array}{l}\text { 8-week head- } \\
\text { to-head trial, } \\
\text { indirect } \\
\text { comparison }\end{array}$ & Univariate & $\begin{array}{l}\text { Escitalopram } \\
\text { dominated }^{\mathrm{a}} \text { citalopram, } \\
\text { fluoxetine and } \\
\text { venlafaxine }\end{array}$ \\
\hline $\begin{array}{l}\text { Francois et al., } \\
2003 \text { [20] } \\
\text { Norway }\end{array}$ & $\begin{array}{l}\text { Escitalopram } \\
\text { vs. } \\
\text { citalopram, } \\
\text { fluoxetine, } \\
\text { venlafaxine }\end{array}$ & $\begin{array}{l}\text { CEA; } \\
\text { decision- } \\
\text { tree }\end{array}$ & $\begin{array}{l}6 \text { months, } \\
\text { societal } \\
\text { perspective }\end{array}$ & $\begin{array}{l}\text { Treatment } \\
\text { success }\end{array}$ & $\begin{array}{l}\text { Remission: MADRS } \leq \\
12\end{array}$ & $\begin{array}{l}\text { 8-week head- } \\
\text { to-head trial, } \\
\text { indirect } \\
\text { comparison }\end{array}$ & Univariate & $\begin{array}{l}\text { Escitalopram } \\
\text { dominated }^{\mathrm{a}} \text { citalopram, } \\
\text { fluoxetine and } \\
\text { venlafaxine }\end{array}$ \\
\hline $\begin{array}{l}\text { Freeman et al., } \\
2000 \text { [21] } \\
\text { UK }\end{array}$ & $\begin{array}{l}\text { Venlafaxine vs. } \\
\text { SSRIs, } \\
\text { TCAs }\end{array}$ & $\begin{array}{l}\text { CEA; } \\
\text { decision- } \\
\text { tree }\end{array}$ & $\begin{array}{l}6 \text { months, } \\
\text { payer } \\
\text { perspective }\end{array}$ & $\begin{array}{l}\text { Treatment } \\
\text { success, }^{f} \\
\text { SFDs }^{f}\end{array}$ & $\begin{array}{l}\text { Response: } 50 \% \\
\text { improve-ment in } \\
\text { HAMD or MADRS }\end{array}$ & Meta-analysis & $\begin{array}{l}\text { Univariate, } \\
\text { probabilistic }\end{array}$ & $\begin{array}{l}\text { Venlafaxine dominated } \\
\text { SSRIs and TCAs }\end{array}$ \\
\hline $\begin{array}{l}\text { Haby et al., } \\
2004 \text { [22] } \\
\text { Australia }\end{array}$ & $\begin{array}{l}\text { CBT vs. } \\
\text { SSRIS }\end{array}$ & $\begin{array}{l}\text { CEA; } \\
\text { decision- } \\
\text { tree }\end{array}$ & $\begin{array}{l}9 \text { months, } \\
\text { payer } \\
\text { perspective }\end{array}$ & DALYs & $\begin{array}{l}\text { Multiple outcomes } \\
\text { averaged for } \\
\text { individual studies }\end{array}$ & Meta-analysis & Probabilistic & $\begin{array}{l}\text { CBT provided by public } \\
\text { psychologist was the } \\
\text { most cost-effective } \\
\text { option }\end{array}$ \\
\hline $\begin{array}{l}\text { Hemels et al., } \\
2004 \text { [23] } \\
\text { Austria }\end{array}$ & $\begin{array}{l}\text { Escitalopram } \\
\text { vs. } \\
\text { citalopram }\end{array}$ & $\begin{array}{l}\text { CEA; } \\
\text { decision- } \\
\text { tree }\end{array}$ & $\begin{array}{l}6 \text { months, } \\
\text { payer and } \\
\text { societal } \\
\text { perspec- } \\
\text { tives }\end{array}$ & $\begin{array}{l}\text { Treatment } \\
\text { success }\end{array}$ & $\begin{array}{l}\text { Remission: MADRS } \leq \\
12\end{array}$ & $\begin{array}{l}\text { 8-week head- } \\
\text { to-head trial }\end{array}$ & $\begin{array}{l}\text { Univariate, } \\
\text { probabilistic }\end{array}$ & $\begin{array}{l}\text { Escitalopram } \\
\text { dominated }^{a} \text { citalopram }\end{array}$ \\
\hline $\begin{array}{l}\text { Howard and } \\
\text { Knight, } 2004 \\
{[24]} \\
\text { Austria }\end{array}$ & $\begin{array}{l}\text { Venlafaxine ER, } \\
\text { venlafaxine IR, } \\
\text { SSRIs }\end{array}$ & $\begin{array}{l}\text { CEA; } \\
\text { decision- } \\
\text { tree }\end{array}$ & $\begin{array}{l}16 \text { week, } \\
\text { payer } \\
\text { perspective }\end{array}$ & $\mathrm{SFDs}^{f}$ & $\begin{array}{l}\text { Remission: not } \\
\text { explicitly reported }\end{array}$ & Meta-analysis & Probabilistic & $\begin{array}{l}\text { Venlafaxine ER was cost- } \\
\text { effective vs. venlafaxine } \\
\text { IR and SSRIs; SSRIs were } \\
\text { least cost-effective }\end{array}$ \\
\hline $\begin{array}{l}\text { Kongsakon } \\
\text { and } \\
\text { Bunchapat- } \\
\text { tanasakda, } \\
2008 \text { [25] } \\
\text { Thailand }\end{array}$ & $\begin{array}{l}\text { Escitalopram } \\
\text { vs. } \\
\text { fluoxetine, } \\
\text { venlafaxine }\end{array}$ & $\begin{array}{l}\text { CEA; } \\
\text { decision- } \\
\text { tree }\end{array}$ & $\begin{array}{l}6 \text { months, } \\
\text { payer and } \\
\text { societal } \\
\text { perspec- } \\
\text { tives }\end{array}$ & $\begin{array}{l}\text { Treatment } \\
\text { success }\end{array}$ & $\begin{array}{l}\text { Remission: MADRS } \leq \\
12\end{array}$ & $\begin{array}{l}2 \text { meta- }^{-} \\
\text {analyses }^{i}\end{array}$ & $\begin{array}{l}\text { Univariate, } \\
\text { probabilistic }\end{array}$ & $\begin{array}{l}\text { Escitalopram } \\
\text { dominated }{ }^{a} \text { fluoxetine } \\
\text { and venlafaxine }\end{array}$ \\
\hline $\begin{array}{l}\text { Kulp et al., } \\
2005 \text { [26] } \\
\text { Germany }\end{array}$ & $\begin{array}{l}\text { Escitalopram } \\
\text { vs. } \\
\text { venlafaxine ER }\end{array}$ & $\begin{array}{l}\text { CEA; } \\
\text { Markov } \\
\text { structure; } \\
\text { 2-week } \\
\text { cycle }\end{array}$ & $\begin{array}{l}70 \text { days, } \\
\text { payer } \\
\text { perspective }\end{array}$ & $\begin{array}{l}\text { Treatment } \\
\text { success }\end{array}$ & $\begin{array}{l}\text { Response: }>50 \% \\
\text { improve-ment in } \\
\text { MADRS } \\
\text { Partial response: } 25- \\
50 \% \\
\text { No response: }<25 \%\end{array}$ & $\begin{array}{l}8 \text { week head- } \\
\text { to-head trial }\end{array}$ & None & $\begin{array}{l}\text { Escitalopram was cost- } \\
\text { effective vs. venlafaxine } \\
\text { ER }\end{array}$ \\
\hline $\begin{array}{l}\text { Lenox-Smith } \\
\text { et al., } 2004 \\
{[27]} \\
\text { UK }\end{array}$ & $\begin{array}{l}\text { Venlafaxine vs. } \\
\text { TCAs, } \\
\text { SSRls } \\
\text { (fluoxetine, } \\
\text { paroxetine, } \\
\text { and } \\
\text { fluvoxamine) }\end{array}$ & $\begin{array}{l}\text { CEA; } \\
\text { decision- } \\
\text { tree }\end{array}$ & $\begin{array}{l}6 \text { months, } \\
\text { payer } \\
\text { perspective }\end{array}$ & $\mathrm{SFDs}^{\dagger}$ & $\begin{array}{l}\text { Remission: } 17- \\
\text { HAMD } \leq 7 \\
\text { Response: } \geq 50 \% \\
\text { improve-ment in } \\
\text { HAMD-21 }\end{array}$ & $\begin{array}{l}\text { Meta-analysis } \\
\text { and a single } \\
\text { study }\end{array}$ & Univariate & $\begin{array}{l}\text { Venlafaxine dominated } \\
\text { SSRIs and TCAs }\end{array}$ \\
\hline $\begin{array}{l}\text { Lenox-Smith } \\
\text { et al., } 2009 \\
\text { [28] } \\
\text { UK }\end{array}$ & $\begin{array}{l}\text { Venlafaxine vs. } \\
\text { fluoxetine, } \\
\text { amitriptyline }\end{array}$ & $\begin{array}{l}\text { CUA; } \\
\text { decision- } \\
\text { tree }\end{array}$ & $\begin{array}{l}6 \text { months, } \\
\text { payer } \\
\text { perspective }\end{array}$ & QALYS & $\begin{array}{l}\text { Remission: HAMD-17 } \\
\leq 7 \\
\text { Response: } \geq 50 \% \\
\text { improve-ment in } \\
\text { HAMD-17 }\end{array}$ & $\begin{array}{l}\text { Pooled data } \\
\text { from } 13 \\
\text { clinical trials }\end{array}$ & Univariate & $\begin{array}{l}\text { Venlafaxine dominated } \\
\text { fluoxetine and } \\
\text { amitriptyline; fluoxetine } \\
\text { dominated }^{\mathrm{a}} \text { amitriptyline }\end{array}$ \\
\hline $\begin{array}{l}\text { Löthgren et } \\
\text { al., } 2004 \text { [29] } \\
\text { Sweden }\end{array}$ & $\begin{array}{l}\text { Escitalopram } \\
\text { vs. } \\
\text { citalopram, } \\
\text { venlafaxine }\end{array}$ & $\begin{array}{l}\text { CEA; } \\
\text { decision- } \\
\text { tree }\end{array}$ & $\begin{array}{l}6 \text { months, } \\
\text { payer and } \\
\text { societal } \\
\text { perspective }\end{array}$ & $\begin{array}{l}\text { Treatment } \\
\text { success }\end{array}$ & $\begin{array}{l}\text { Remission: MADRS } \leq \\
12\end{array}$ & Meta-analysis & $\begin{array}{l}\text { Univariate, } \\
\text { probabilistic }\end{array}$ & $\begin{array}{l}\text { Escitalopram } \\
\text { dominated }^{a} \text { citalopram } \\
\text { and venlafaxine }\end{array}$ \\
\hline $\begin{array}{l}\text { Machado et } \\
\text { al., } 2007 \text { [30] } \\
\text { Brazil }\end{array}$ & $\begin{array}{l}\text { SNRIS vS. SSRIS, } \\
\text { TCAS }\end{array}$ & $\begin{array}{l}\text { CEA; } \\
\text { decision- } \\
\text { tree }\end{array}$ & $\begin{array}{l}6 \text { months, } \\
\text { payer } \\
\text { perspective }\end{array}$ & $\begin{array}{l}\text { Treatment } \\
\text { success }\end{array}$ & $\begin{array}{l}\text { Remission: score } \leq 7 \\
\text { on HAMD or } \leq 12 \\
\text { on MADRS }\end{array}$ & Meta-analysis & $\begin{array}{l}\text { Univariate, } \\
\text { probabilistic }\end{array}$ & $\begin{array}{l}\text { SNRIs dominated }{ }^{a} \text { SSRIs } \\
\text { and TCAs }\end{array}$ \\
\hline
\end{tabular}


Table 1 Summary of methods and conclusions of included studies (Continued)

\begin{tabular}{|c|c|c|c|c|c|c|c|c|}
\hline $\begin{array}{l}\text { Malone, } 2007 \\
\text { [31] } \\
\text { US }\end{array}$ & $\begin{array}{l}\text { SSRIS, } \\
\text { escitalopram, } \\
\text { paroxetine CR, } \\
\text { sertraline, } \\
\text { venlafaxine ER }\end{array}$ & $\begin{array}{l}\text { CEA; } \\
\text { structure } \\
\text { not } \\
\text { explicitly } \\
\text { reported }\end{array}$ & $\begin{array}{l}6 \text { months, } \\
\text { payer } \\
\text { perspective }\end{array}$ & $\begin{array}{l}\text { Treatment } \\
\text { success }\end{array}$ & $\begin{array}{l}\text { Response: } \geq 50 \% \\
\text { improve-ment in } \\
\text { HAMD or MADRS } \\
\text { Remission: HAMD } \leq \\
7 \text { or MADRS } \leq 10\end{array}$ & $\begin{array}{l}\text { Pooled } \\
\text { analysis of } \\
\text { trials }\end{array}$ & $\begin{array}{l}\text { Univariate, } \\
\text { probabilistic }\end{array}$ & $\begin{array}{l}\text { Venlafaxine had the } \\
\text { lowest ICER followed by } \\
\text { escitalopram and } \\
\text { sertraline; paroxetine } \\
\text { was dominated }\end{array}$ \\
\hline $\begin{array}{l}\text { Nuijten, } 2001 \\
\text { [32] } \\
\text { The } \\
\text { Netherlands }\end{array}$ & $\begin{array}{l}\text { Prolongation } \\
\text { of } \\
\text { antidepressant } \\
\text { medication vs. } \\
\text { no } \\
\text { prolongation }\end{array}$ & $\begin{array}{l}\text { CUA; } \\
\text { Markov } \\
\text { structure; } \\
\text { 8-week } \\
\text { cycle }\end{array}$ & $\begin{array}{l}9 \text { months, } \\
\text { payer and } \\
\text { societal } \\
\text { perspec- } \\
\text { tives }\end{array}$ & $\begin{array}{l}\text { QALYS, } \\
\text { TWD }\end{array}$ & $\begin{array}{l}\text { Not explicitly } \\
\text { reported }\end{array}$ & $\begin{array}{l}\text { Published } \\
\text { literature }\end{array}$ & Univariate & $\begin{array}{l}\text { Continuation treatment } \\
\text { was not cost-effective, } \\
\text { unless extended to } \\
\text { maintenance }\end{array}$ \\
\hline $\begin{array}{l}\text { Perlis et al., } \\
2009 \text { [33] } \\
\text { US }\end{array}$ & $\begin{array}{l}\text { Test for SSRI } \\
\text { responsive- } \\
\text { ness vs. } \\
\text { no test }\end{array}$ & $\begin{array}{l}\text { CUA; } \\
\text { Markov } \\
\text { structure; } \\
\text { 3-month } \\
\text { cycle }\end{array}$ & $\begin{array}{l}3 \text { years, } \\
\text { societal } \\
\text { perspective }\end{array}$ & QALYS & $\begin{array}{l}\text { Remission: } \\
\text { Instrument not } \\
\text { explicitly reported }\end{array}$ & $\begin{array}{l}\operatorname{STAR}^{*} \mathrm{D} \text { trial } \\
{[34]}\end{array}$ & $\begin{array}{l}\text { Univariate, } \\
\text { two-way }\end{array}$ & $\begin{array}{l}\text { The ICER for the genetic } \\
\text { test would not be } \\
\text { considered cost effective }\end{array}$ \\
\hline $\begin{array}{l}\text { Sado et al., } \\
2009 \text { [35] } \\
\text { Japan }\end{array}$ & $\begin{array}{l}\text { COMBI vs. } \\
A D\end{array}$ & $\begin{array}{l}\text { CUA; } \\
\text { decision- } \\
\text { tree }\end{array}$ & $\begin{array}{l}12 \text { months, } \\
\text { payer and } \\
\text { societal } \\
\text { perspec- } \\
\text { tives }\end{array}$ & $\begin{array}{l}\text { Treatment } \\
\text { success, } \\
\text { QALYs }\end{array}$ & $\begin{array}{l}\text { No response: HRSD- } \\
17>6 \text { or HRSD- } 24 \\
>8\end{array}$ & $\begin{array}{l}\text { Meta-analysis } \\
\text { of } 8 \text { RCTs }\end{array}$ & $\begin{array}{l}\text { Univariate, } \\
\text { probabilistic }\end{array}$ & $\begin{array}{l}\text { COMBI was cost- } \\
\text { effective }\end{array}$ \\
\hline $\begin{array}{l}\text { Simon et al., } \\
2006[36] \\
\text { UK }\end{array}$ & $\begin{array}{l}\text { COMBI vs. } \\
\text { AD }\end{array}$ & $\begin{array}{l}\text { CUA; } \\
\text { decision- } \\
\text { tree }\end{array}$ & $\begin{array}{l}15 \text { months, } \\
\text { payer } \\
\text { perspective }\end{array}$ & $\begin{array}{l}\text { Treatment } \\
\text { success, } \\
\text { QALYs }\end{array}$ & $\begin{array}{l}\text { Remission: HRSD-17 } \\
\leq 6 \text { or HRSD- } 24 \leq 8\end{array}$ & Meta-analysis & $\begin{array}{l}\text { Univariate, } \\
\text { probabilistic }\end{array}$ & $\begin{array}{l}\text { COMBI was cost- } \\
\text { effective }\end{array}$ \\
\hline $\begin{array}{l}\text { Sobocki et al., } \\
2008[37] \\
\text { Sweden }\end{array}$ & $\begin{array}{l}\text { Venlafaxine } \\
\text { maintenance } \\
\text { treatment vs. } \\
\text { placebo }\end{array}$ & $\begin{array}{l}\text { CUA; } \\
\text { Markov } \\
\text { structure; } \\
\text { 1-month } \\
\text { cycle }\end{array}$ & $\begin{array}{l}2 \text { years, } \\
\text { payer and } \\
\text { societal } \\
\text { perspec- } \\
\text { tives }\end{array}$ & QALYS & $\begin{array}{l}\text { Time to recurrence: } \\
17-\mathrm{HAMD}>12 \text { and } \\
\geq 50 \% \text { improve- } \\
\text { ment in } 17-\mathrm{HAMD}\end{array}$ & 2-year trial & $\begin{array}{l}\text { Univariate, } \\
\text { probabilistic }\end{array}$ & $\begin{array}{l}\text { Maintenance treatment } \\
\text { with venlafaxine was } \\
\text { cost-effective }\end{array}$ \\
\hline $\begin{array}{l}\text { Sorenson et } \\
\text { al., } 2007 \text { [38] } \\
\text { Denmark }\end{array}$ & $\begin{array}{l}\text { Escitalopram } \\
\text { vs. } \\
\text { citalopram, } \\
\text { venlafaxine ER }\end{array}$ & $\begin{array}{l}\text { CEA; } \\
\text { decision- } \\
\text { tree }\end{array}$ & $\begin{array}{l}6 \text { months, } \\
\text { payer and } \\
\text { societal } \\
\text { perspec- } \\
\text { tives }\end{array}$ & $\begin{array}{l}\text { Treatment } \\
\text { success }\end{array}$ & $\begin{array}{l}\text { Remission: MADRS } \leq \\
12\end{array}$ & Meta-analysis & $\begin{array}{l}\text { Univariate, } \\
\text { probabilistic }\end{array}$ & $\begin{array}{l}\text { Escitalopram } \\
\text { dominated }^{a} \text { citalopram; } \\
\text { similar cost-effectiveness } \\
\text { vs. venlafaxine ER }\end{array}$ \\
\hline $\begin{array}{l}\text { Sullivan et al., } \\
2004 \text { [39] } \\
\text { US }\end{array}$ & $\begin{array}{l}\text { Escitalopram, } \\
\text { citalopram, } \\
\text { fluoxetine, } \\
\text { venlafaxine ER, } \\
\text { sertraline, } \\
\text { paroxetine, } \\
\text { paroxetine CR, } \\
\text { venlafaxine }\end{array}$ & $\begin{array}{l}\text { CUA; } \\
\text { decision- } \\
\text { tree }\end{array}$ & $\begin{array}{l}6 \text { months, } \\
\text { payer } \\
\text { perspective }\end{array}$ & QALYS & $\begin{array}{l}\text { Response: }>50 \% \\
\text { improve-ment in } \\
\text { MADRS (with } \\
\text { treatment } \\
\text { maintained for } \geq \\
180 \text { days) }\end{array}$ & $N / A^{l}$ & $\begin{array}{l}\text { Univariate, } \\
\text { probabilistic }\end{array}$ & $\begin{array}{l}\text { Escitalopram } \\
\text { dominated }^{a} \text { all } \\
\text { treatments }^{m}\end{array}$ \\
\hline $\begin{array}{l}\text { Trivedi et al., } \\
2004 \text { [40] } \\
\text { US }\end{array}$ & $\begin{array}{l}\text { Venlafaxine ER } \\
\text { vs. } \\
\text { SSRIs } \\
\text { (fluvoxamine, } \\
\text { fluoxetine, } \\
\text { paroxetine) }\end{array}$ & $\begin{array}{l}\text { CUA; } \\
\text { decision- } \\
\text { tree }\end{array}$ & $\begin{array}{l}8 \text { weeks, } \\
\text { payer } \\
\text { perspective }\end{array}$ & $\begin{array}{l}\text { QADs and } \\
\text { DFDs }\end{array}$ & $\begin{array}{l}\text { Response: HAMD < } \\
15 \text { and/or } \geq 50 \% \\
\text { improve-ment in } \\
\text { HAMD. Remission: } \\
\text { HAMD } \leq 7 \\
\text { Response without } \\
\text { remission: HAMD 8- } \\
14\end{array}$ & $\begin{array}{l}\text { Pooled } \\
\text { analysis of } 8 \\
\text { RCTs }\end{array}$ & Probabilistic & $\begin{array}{l}\text { Venlafaxine ER was cost- } \\
\text { effective vs. SSRIs }\end{array}$ \\
\hline $\begin{array}{l}\text { van Baardewijk } \\
\text { et al., } 2005 \\
\text { [41] } \\
\text { Canada }\end{array}$ & $\begin{array}{l}\text { Duloxetine vs. } \\
\text { venlafaxine ER }\end{array}$ & $\begin{array}{l}\text { CEA; } \\
\text { decision- } \\
\text { tree }\end{array}$ & $\begin{array}{l}6 \text { months, } \\
\text { payer and } \\
\text { societal } \\
\text { perspec- } \\
\text { tives }\end{array}$ & $\begin{array}{l}\text { Treatment } \\
\text { success, } \\
\text { SFDs }\end{array}$ & $\begin{array}{l}\text { Remission: } \mathrm{HAMD} \leq \\
7 \text { or } \mathrm{MADRS} \leq 10\end{array}$ & Meta-analysis & $\begin{array}{l}\text { Univariate, } \\
\text { probabilistic }\end{array}$ & $\begin{array}{l}\text { Venlafaxine ER } \\
\text { dominated }^{a} \text { duloxetine }\end{array}$ \\
\hline $\begin{array}{l}\text { Vos et al., } \\
2005 \text { [42] } \\
\text { Australia }\end{array}$ & $\begin{array}{l}\text { Listed in } \\
\text { footnote }^{n}\end{array}$ & $\begin{array}{l}\text { CEA; } \\
\text { decision- } \\
\text { tree }\end{array}$ & $\begin{array}{l}9 \text { months } \\
\text { and } 5 \text { years, } \\
\text { payer } \\
\text { perspective }\end{array}$ & DALYS & Multiple outcomes & $\begin{array}{l}\text { Meta- } \\
\text { analyses }\end{array}$ & Probabilistic & $\begin{array}{l}\text { All interventions had a } \\
\text { favourable ICER under } \\
\text { Australian health service } \\
\text { conditions }\end{array}$ \\
\hline $\begin{array}{l}\text { Wade et al., } \\
2005 \text { [43] } \\
\text { UK }\end{array}$ & $\begin{array}{l}\text { Escitalopram } \\
\text { vs. } \\
\text { citalopram, } \\
\text { venlafaxine }\end{array}$ & $\begin{array}{l}\text { CEA; } \\
\text { decision- } \\
\text { tree }\end{array}$ & $\begin{array}{l}6 \text { months, } \\
\text { payer and } \\
\text { societal } \\
\text { perspective }\end{array}$ & $\begin{array}{l}\text { Treatment } \\
\text { success }\end{array}$ & $\begin{array}{l}\text { Remission: MADRS } \leq \\
12\end{array}$ & Meta-analysis & $\begin{array}{l}\text { Univariate, } \\
\text { probabilistic }\end{array}$ & $\begin{array}{l}\text { Escitalopram } \\
\text { dominated }^{\text {a }} \text { citalopram; } \\
\text { similar cost-effectiveness } \\
\text { vs. venlafaxine }\end{array}$ \\
\hline
\end{tabular}


Table 1 Summary of methods and conclusions of included studies (Continued)

\begin{tabular}{|c|c|c|c|c|c|c|c|c|}
\hline $\begin{array}{l}\text { Wade et al., } \\
2005[44] \\
\text { UK }\end{array}$ & $\begin{array}{l}\text { Escitalopram } \\
\text { vs. } \\
\text { citalopram }\end{array}$ & $\begin{array}{l}\text { CEA; } \\
\text { decision- } \\
\text { tree }\end{array}$ & $\begin{array}{l}6 \text { months, } \\
\text { payer and } \\
\text { societal } \\
\text { perspec- } \\
\text { tives }\end{array}$ & $\begin{array}{l}\text { Treatment } \\
\text { success }\end{array}$ & $\begin{array}{l}\text { Remission: MADRS } \leq \\
12 \\
\text { Response: } \geq 50 \% \\
\text { improve-ment in } \\
\text { MADRS }\end{array}$ & Meta-analysis & $\begin{array}{l}\text { Univariate, } \\
\text { probabilistic }\end{array}$ & $\begin{array}{l}\text { Escitalopram } \\
\text { dominated }^{a} \text { citalopram }\end{array}$ \\
\hline $\begin{array}{l}\text { Xie et al., } 2009 \\
{[45]} \\
\text { Singapore }\end{array}$ & $\begin{array}{l}\text { Escitalopram } \\
\text { vs. } \\
\text { venlafaxine, } \\
\text { fluvoxamine }\end{array}$ & $\begin{array}{l}\text { CEA; } \\
\text { decision- } \\
\text { tree }\end{array}$ & $\begin{array}{l}6 \text { months, } \\
\text { societal } \\
\text { perspective }\end{array}$ & $\begin{array}{l}\text { Treatment } \\
\text { success }\end{array}$ & $\begin{array}{l}\text { Remission: MADRS } \\
\text { score } \leq 12\end{array}$ & $\begin{array}{l}\text { Head-to-head } \\
\text { trials }^{\circ}\end{array}$ & $\begin{array}{l}\text { Univariate, } \\
\text { probabilistic }\end{array}$ & $\begin{array}{l}\text { Escitalopram } \\
\text { dominated }^{a} \text { venlafaxine } \\
\text { and fluvoxamine }\end{array}$ \\
\hline
\end{tabular}

$\mathrm{AD}=$ antidepressant therapy; $\mathrm{CEA}=$ cost-effectiveness analysis; $\mathrm{CBT}=$ cognitive behavioural therapy; $\mathrm{CGI}=\mathrm{Clinical}$ Global Impression; COMBI = combination therapy; $\mathrm{CR}=$ controlled release; $\mathrm{CUA}=$ cost-utility analysis; $\mathrm{DALY}=$ disability-adjusted life-years; $\mathrm{DFD}=$ disease-free day; $\mathrm{ER}=$ extended release; $\mathrm{HAMD}=$ Hamilton Depression Rating Scale; HDRS = Hamilton Depression Rating Scale; HRSD = Hamilton Rating Scale for Depression; ICER = incremental cost-effectivenesS ratio; IR = instant release; MADRS = Montgomery Åsberg Depression Rating Scale; MDD = major depressive disorder; MECT = maintenance electroconvulsive therapy; MPT = maintenance pharmacotherapy; N/A = not applicable; $\mathrm{QAD}=$ quality-adjusted day; $\mathrm{QALW}=$ quality-adjusted life week; QALY = quality-adjusted life-year; RCT = randomised controlled trial; SFD = symptom-free day; SNRI = serotonin-norepinephrine reuptake inhibitor; SSRI = selective serotonin reuptake inhibitor; STAR*D = Sequenced Treatment Alternatives to Relieve Depression trial; TCA = tricyclic antidepressants; TWD = time without depression; UK = United Kingdom; US = United States; WTP = willingness to pay.

${ }^{a}$ A dominant therapy is less expensive and more effective than the comparator.

${ }^{b}$ MPT is compared with MECT in elderly individuals with MDD who relapsed after responding to initial course of electroconvulsive therapy.

c Included 6 placebo-controlled RCTs with SSRIs as active arm comparators and 2 head-to-head venlafaxine trials; a meta-analysis was used to obtain response and remission rates for mirtazapine.

${ }^{d}$ In the absence of efficacy data for mirtazapine in patients with more severe illness, mirtazapine rates were calculated by applying the mean differences between the less severe and the more severe population to the mirtazapine rates reported in the meta-analysis for the patient population with HAMD-17 $\geq 18$. e Germany, Italy, Netherlands, Poland, Spain, Sweden, Switzerland, UK, US, Venezuela.

$\mathrm{f}$ SFDs were measured as time elapsed after the determination of success through the end of the period being analysed.

${ }^{g}$ Continuous treatment with milnacipran.

${ }^{\mathrm{h}}$ Follow-up with no preventive treatment.

' Due to lack of published data on head-to-head comparison of escitalopram and fluoxetine, the clinical data comparing escitalopram to citalopram were used as a proxy, derived from a published meta-analysis.

${ }^{\mathrm{j}}$ ICERs were calculated using SSRIs as the reference group.

${ }^{k}$ A therapy dominated by other comparators has higher cost and lower effectiveness.

' Due to a lack of consistent and comprehensive studies demonstrating differences in efficacy across all 8 serotonin reuptake inhibitors, and in order to retain a specific focus on the impact of adverse drug reactions on treatment costs, it was assumed that on average $60 \%$ of patients respond to serotonin reuptake inhibitor therapy.

${ }^{m}$ Escitalopram had lowest direct costs and the greatest effectiveness, followed by citalopram, generic fluoxetine, venlafaxine ER, sertraline, generic paroxetine, paroxetine $C R$, and venlafaxine IR.

n SSRIs (acute, continuation, maintenance), TCAs (acute, continuation, maintenance), Bibliotherapy (acute), Acute and maintenance: individual CBT public psychologist, individual CBT, private psychologist, individual CBT public psychiatrist, individual CBT private psychiatrist, group CBT public psychologist.

${ }^{\circ}$ Due to lack of data on head-to-head comparisons of escitalopram and fluvoxamine, a head-to-head comparison of citalopram and fluvoxamine was used as a proxy.

covered 10 countries (Germany, Italy, Netherlands, Poland, Spain, Sweden, Switzerland, UK, US, and Venezuela) and performed a clinical management analysis to estimate the country-specific treatment options and outcomes within the set structure of the decision-tree model in Figure 3. Lenox-Smith and colleagues [27,28] amended the structure by including an extra arm representing a clinical outcome node where patient could experience improvement without achieving remission.

The main difference in the model structures reported by Casciano and colleagues [15] and Francois and colleagues [19] is that the latter structure included two paths, one for primary care and one for secondary care. Another difference is that these models incorporated different options for patients failing first and second lines of treatment. The Francois structure offered the option of hospitalisation once all treatment options have been exhausted. For patients experiencing remission, the Casciano structure assumed continuation on treatment for 6 months, whereas the Francois structure incorporated a risk of premature treatment discontinuation. Unlike models based on the structure reported in Francois [19], models adapting the structure by Casciano [15] did not incorporate rates of suicide or suicide attempts.

The systematic review identified 8 models $[9-11,16,26,32,33,37]$ that were described as having a Markov structure. Time horizons ranged from 70 days [26] to lifetime [10], longer than the time horizons in decision-tree models. The cycle lengths of the Markov models ranged from 1 week [9] to 6 months [10]. A variety of health states were defined. In the model by Aziz and colleagues [10], health states were wellness (full remission), partial depression (partial remission), depression (no response), death by suicide, or death by other cause. Benedict and colleagues [11] included relapse, recurrence, and treatment switches. Dardennes and colleagues [16] distinguished between remission with follow-up and remission without follow-up. The model by Perlis and colleagues [33] simply used 

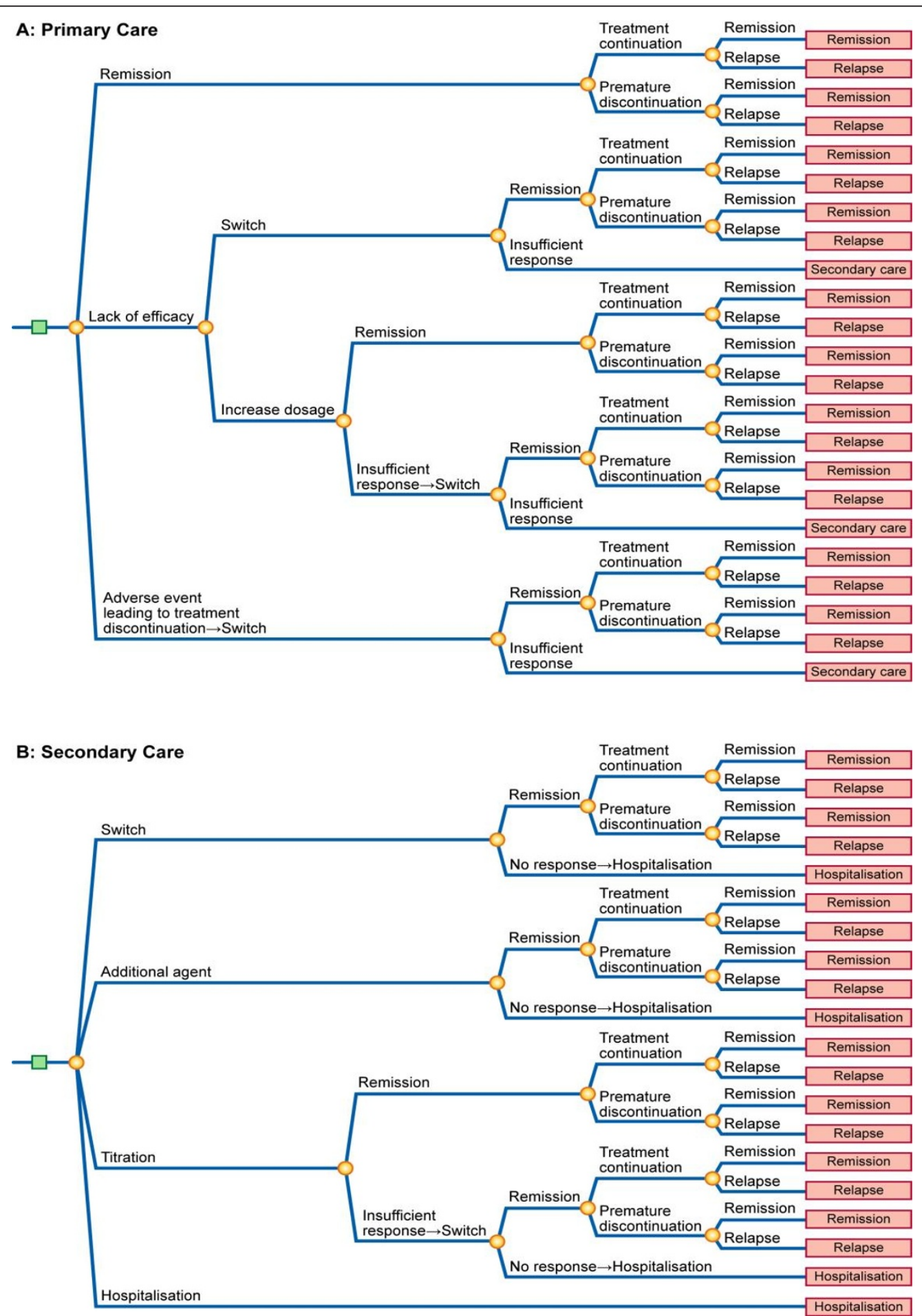

Figure 2 Two-path model structure reported by Francois et al., 2002. Reproduced from Francois et al. (2002) [19]. 


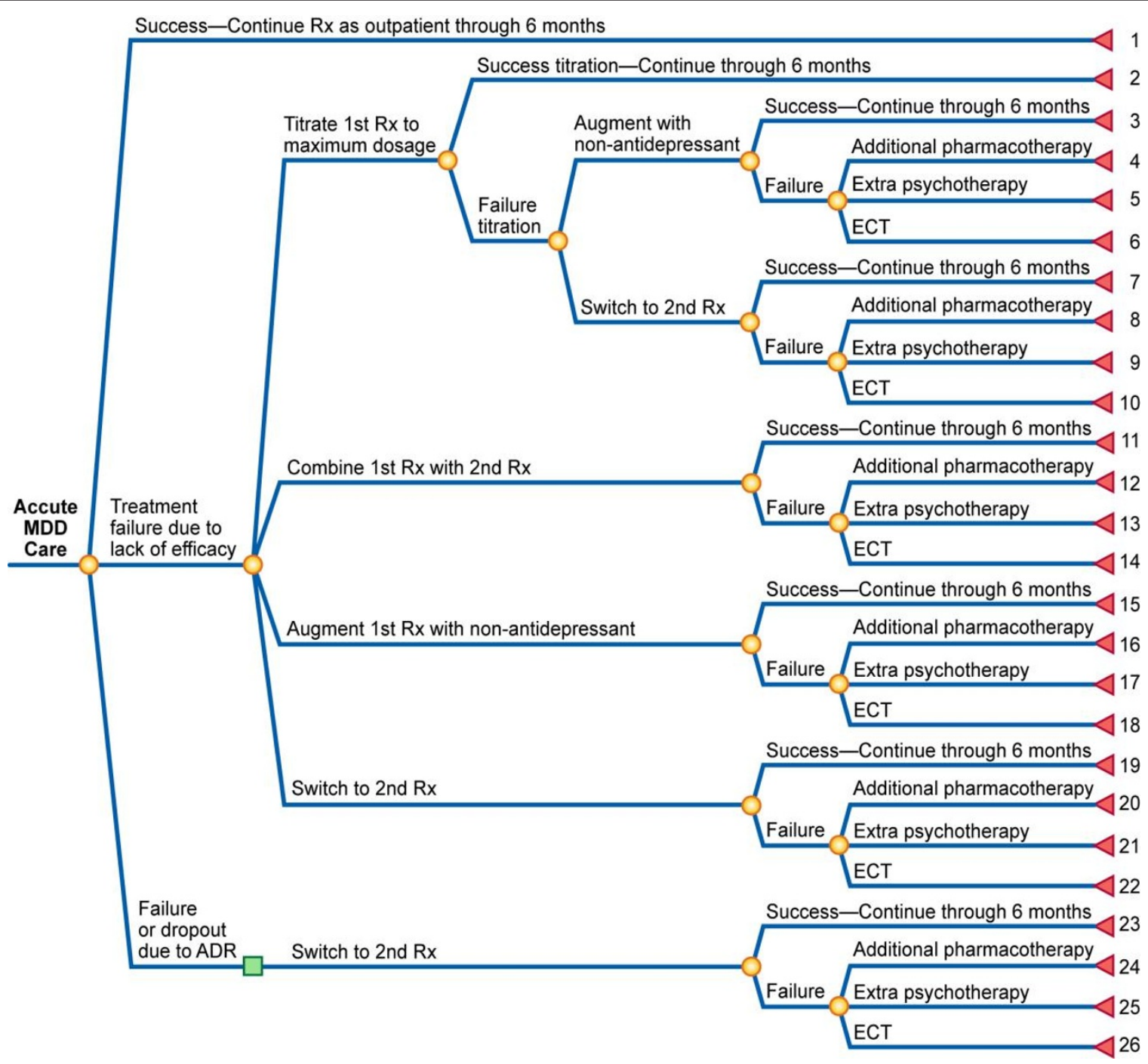

Figure 3 Model structure reported by Casciano et al., 2000. ADR = adverse drug reaction; $E C T=$ electroconvulsive therapy; $R x=$ prescription. Reproduced from Casciano et al. (2000) [15]. Image reprinted with permission from Medscape.com, 2010. Available at: http://www. medscape.com/viewarticle/409930.

"depressed" (on or off therapy) and "well" (on or off therapy).

\section{Model time horizon}

A number of economic evaluation guidelines state that the model time horizon is dependent upon the time at which full benefits of the studied intervention can be realised $[47,48]$. With treatment of depression, certain treatment benefit can be realised over a shorter or a longer time horizon, depending on the treatment stage (Figure 4) [49].

The treatment phases include acute treatment, during which time the goal is to resolve symptoms; continuation treatment, during which time therapy is continued to ensure complete resolution of the index episode and to prevent relapse; and long-term maintenance, during which time optimal therapy is continued to prevent the development of a new episode [50]. Clinical trials in MDD are often conducted over a period of a few weeks, typically 6 to 8 weeks [51], representing the acute phase of a depressive disorder. All three treatment phases should be implemented to optimise treatment outcome [49]. If no improvement is observed after a few weeks of medication, or if undesirable adverse events have occurred, it is recommended to consider titration of the prescribed dose or switch to a different antidepressant. Where improvement is observed, it is recommended to continue the medication until the underlying depression has disappeared [38]. The majority of the identified models had a time horizon of 6 months, covering the 


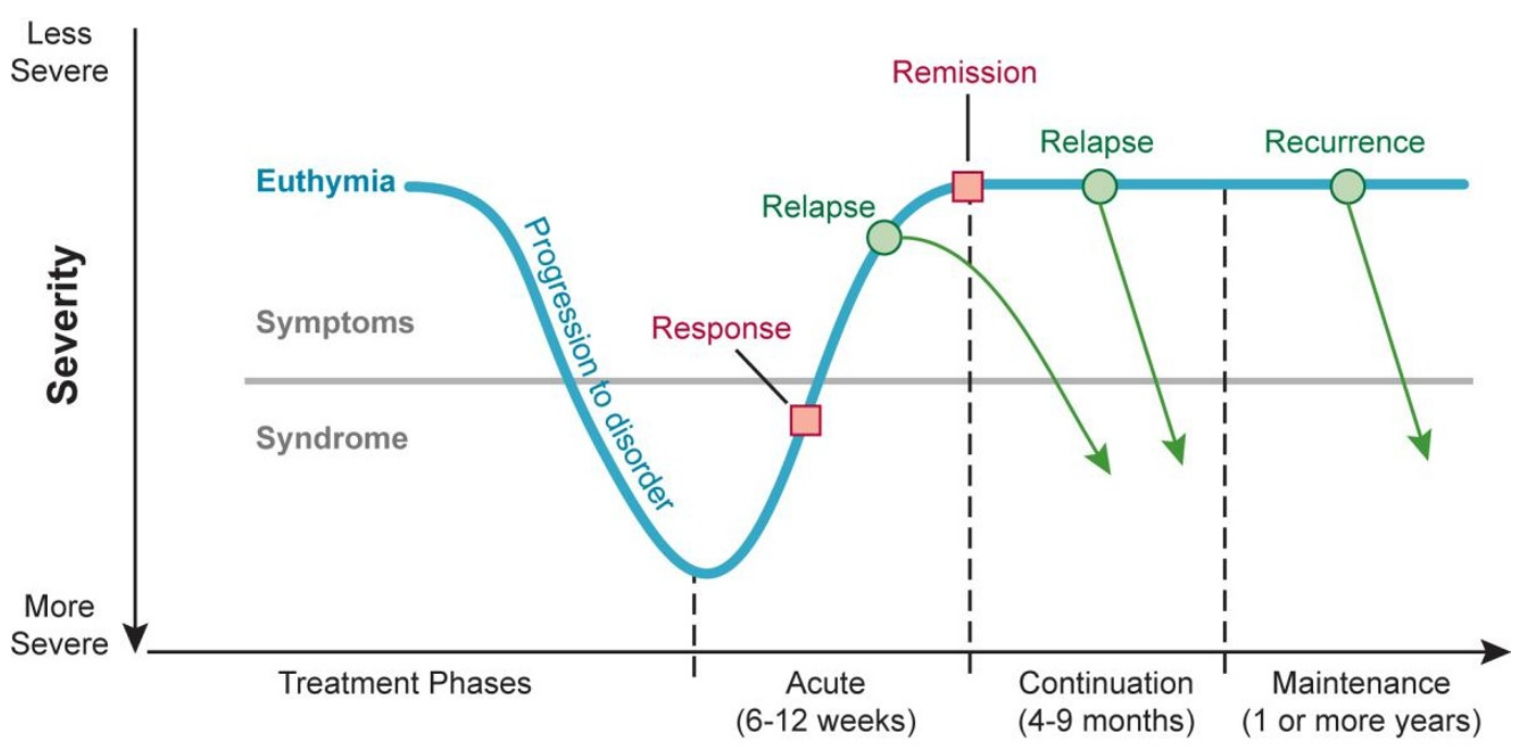

Time

Figure 4 Phases of treatment for MDD. MDD = major depressive disorder. Adapted, with permission, from Bakish et al. [49].

acute and continuation phases, or the length of time that patients with a first episode of MDD should normally be treated [27]. Three studies adopted a time horizon of less than 6 months $[24,26,40]$, and three models used a time horizon of 1 year $[9,16,35]$; other time horizons applied in the identified models included lifetime [10], 3 years [33], 2 years [37], and 15 months [36].

\section{Patient population}

The majority of the models were constructed in a population of adults with MDD who were starting initial therapy, with one study in children and adolescents [22]. No studies were identified in patients with MDD experiencing partial response to initial therapy. Two studies were in patients who did not respond to initial therapy [11,31], two studies were in patients with recurrent depression [16,37], one study was in elderly patients who responded to a course of ECT but then relapsed [10].

\section{Model comparators}

The comparisons of MDD treatments evaluated in 30 of the included models focusing on acute and continuation phases are presented in Figure 5. Venlafaxine and escitalopram are the most intensively studied interventions, evaluated in 21 and 15 studies, respectively; followed by SSRIs as a drug class, which were examined in 10 studies, and TCAs as a drug class, which were compared in 6 studies. A number of studies (7 of 37) included treatment evaluations during the maintenance phase.
Sobocki and colleagues [37] evaluated venlafaxine maintenance treatment versus placebo. One model [35] compared combination therapy with antidepressant therapy alone. Aziz and colleagues [10] examined maintenance pharmacotherapy versus maintenance ECT. Dardennes and colleagues [16] compared preventative strategy (i.e., a maintenance treatment with milnacipran) and episodic strategy (i.e., medical follow-up treating new episodes when diagnosed). One study [42] included multiple comparisons of treatments in acute and continuation phases (SSRIs, TCAs, CBT, and bibliotherapy) and in maintenance phase (TCAs, SSRIs, and various CBT options). Perlis and colleagues [33] assessed the costeffectiveness of a pharmacogenetic test for SSRI responsiveness versus a 'no-test' condition. Nuijten [32] assessed the cost-effectiveness of continuation treatment with SSRIs compared with no preventative treatment and examined the impact of extending the continuation treatment to maintenance treatment in a scenario analysis.

\section{Efficacy and safety data}

Both response and remission rates were applied in the economic models as measures of treatment success. However, the definitions of remission and response were not applied consistently across the studies.

Response was most commonly defined as a $50 \%$ or greater improvement in the Montgomery Asberg Depression Rating Scale (MADRS) [52] score or the Hamilton Rating Scale for Depression (HAMD-17) score 


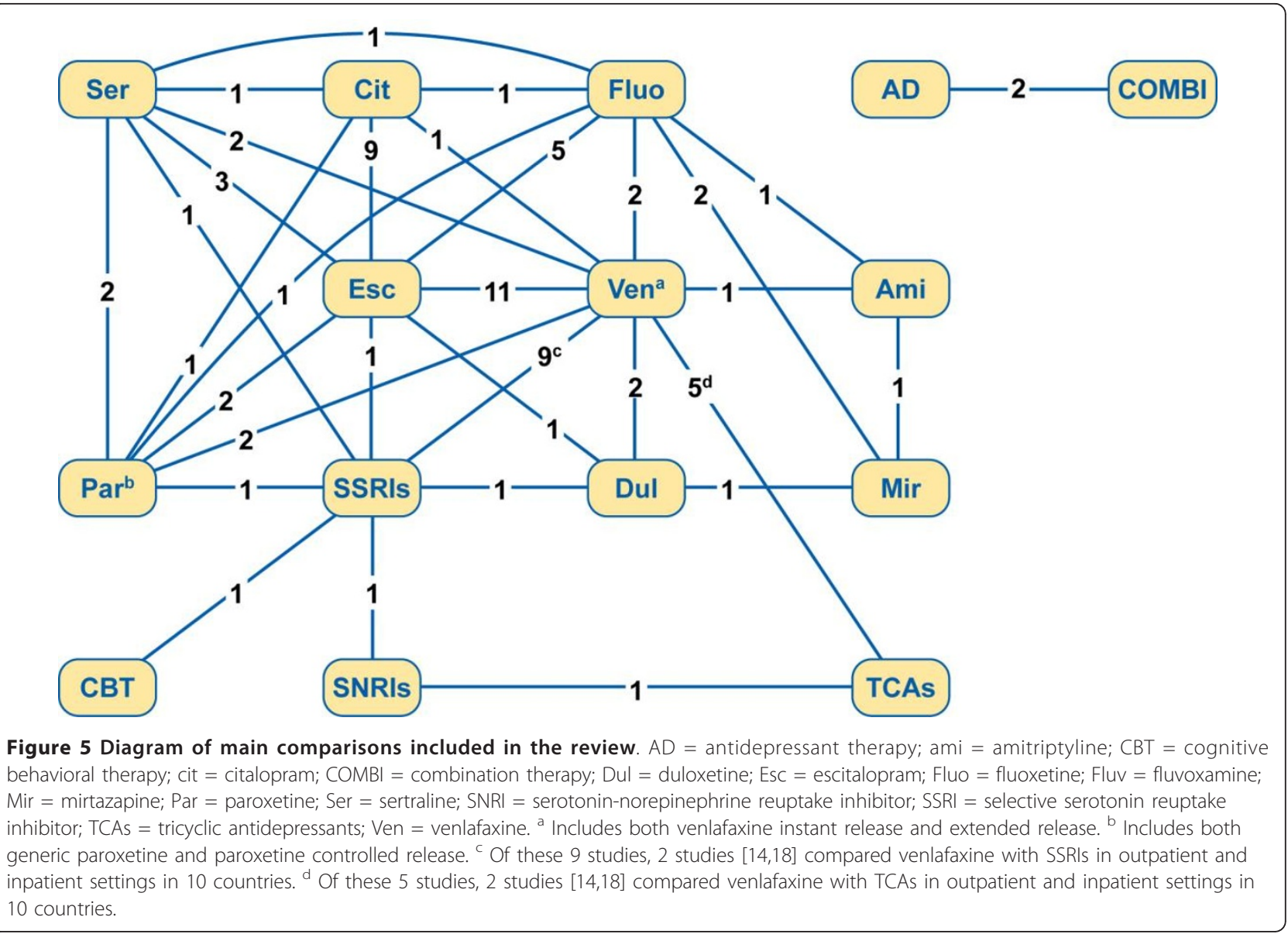

[53]. One study [13] defined response as a $50 \%$ or greater improvement in the 21-item HAMD score or a score of 1 or 2 on the patient-rated Clinical Global Impression scale [54]. One study used non-response, defined as a score greater than 6 on the HAMD-17 scale (or greater than 8 on the HAMD-24). One model [26] used three definitions for varying levels of response based on MADRS scale: response (greater than 50\% improvement from baseline), partial response (25\%-50\% improvement from baseline) and no response $(<25 \%$ improvement from baseline).

General consensus suggests that values of 7 or less on the HAMD are indicative of clinical remission; for the MADRS instrument, many clinicians have come to accept that values of 10 or less are likely to indicate remission [31]. From the identified models, five studies $[9,12,27,28,40]$ used clinical data that defined remission as a score of 7 or less on HAMD, 10 studies $[17,19,20,23,25,29,38,43-45]$ incorporated remission defined as score of 12 or less on MADRS, two studies $[9,30]$ used both of these definitions, and one study [36] defined remission as a score of 8 or less on the HAMD24 or a score of 6 or less on the HAMD-17. Out of 14 models using the MADRS-based definition of remission, only two $[31,41]$ used a cut-off value of $\leq 10$, with the majority of the models using a cut-off value of $\leq 12$.

The variability in the definitions of remission and response applied across the studies presents challenges in performing indirect comparisons of interventions where no head-to-head studies exist. As a result, the majority of studies derived primary clinical inputs from single trials $[8,13,16,23,26,33,37,45]$ or via simple pooling of data from multiple trials $[9,28,31,40]$, or a combination of single trials, pooled analyses, indirect comparisons and meta-analyses [11,12,18-20,25,27]. A fair amount of models (15 of 37) used meta-analyses to obtain primary efficacy inputs $[14,15,17,21,22,24,29$, $30,35,36,38,41-44$ ]. The majority of models (26 of 37) incorporated adverse events; 23 models included adverse events as an outcome leading to treatment discontinuation; only 3 studies $[8,9,39]$ modelled the impact of individual adverse events in terms of utility and costs. Efficacy data applied after change of treatment were most commonly obtained from expert opinion or published literature. Duration of trials used in the models as sources of clinical data were typically between 6 and 12 weeks. 


\section{Resource use and costs}

Resource use and cost estimates used in the identified analyses were predominantly obtained and/or validated by expert opinion (22 of 37). Malone and colleagues [31] applied 6-month resource use and costs estimates from a retrospective analysis of accounting records of 1,814 patients enrolled in nine randomised, controlled trials [55]. Sobocki and colleagues [37] used cost data from the naturalistic observational study Health Economic Aspects of Depression in Sweden, conducted in Swedish primary care [56]. For primary care, Wade and colleagues [43] used resource use and cost estimates from the UK General Practice Research Database analysis. Two studies $[33,39]$ used prospective cohort studies in estimating resource use and costs.

The majority of studies employing a payer perspective included medication costs, costs of physicians' time, diagnostic and monitoring tests, hospitalisation and psychotherapy. The specific resources applied varied substantially between the studies. Only a few studies reported the costs of managing adverse events, which were included in the base-case analyses [8,9,12,24,25,39]. A number of studies [10,16,17,19,20,23,25,29,33,43,44] reported the cost of suicide and/or suicide attempt.

Almost half of the identified studies conducted their analyses from the societal perspective. The majority of studies estimated indirect costs associated with productivity losses using the Human Capital approach $[11,19,20,23,25,29,38,41,43-45]$, and a few used the Friction Costs approach $[17,32]$. The US study in elderly patients with recurrent MDD also included costs associated with lost leisure time activities and wages lost by caregivers [10].

\section{Health state utilities}

Of the identified models, 14 included utility weight estimates to calculate quality-adjusted life-years (QALYs). The utility values applied in the models varied across the same health states by between 0.11 and 0.21 , suggesting that the utility weights applied in the models were not consistent (Table 2) [57-68]. Revicki and Wood [62] was the most commonly cited source of utility values applied in the models. This study used the HAMD, SF-36 Health Survey, and standard gamble interviews to obtain utilities for 11 hypothetical depression-related states varying by depression severity (i.e., mild, moderate, severe), medication (i.e., nefazodone, fluoxetine, imipramine), and treatment status (i.e., maintenance treatment or no treatment). The mean utility for severe, untreated depression was the lowest, 0.30 . The highest mean utility was for remission without treatment, 0.86. Medication-specific utilities varied from 0.55 to 0.63 for moderate depression, 0.64 to 0.73 for mild depression, and 0.72 to 0.83 for antidepressant remission maintenance therapy [62]. The study by Simon and colleagues [36] used these estimates in the base case analysis, and in the sensitivity analysis the study investigated the effect of partial response to treatment using uncertainty ranges of 0.30 to 0.63 and 0.63 to 0.70 for severe and moderate depression, respectively. The study highlighted the scarcity of evidence on the health-related quality of life of people with depression.

Benedict and colleagues [11] used utilities derived from the EuroQol Five Dimension instrument [69] scores of approximately 300 European patients representing the head-to-head clinical trial population. The model used utility values of 0.79 for remitters, 0.68 for responders, 0.55 for non-responders, and 0.53 for those dropping out. Utility of patients achieving remission and staying in remission without treatment (0.86) was obtained from Revicki and Wood [62]. Another UK model [28] used the methodology by Lave and colleagues [68] to transform the disease-free days (DFDs) into utility weights. The number of DFDs between measurements over any given interval was first estimated by adding the calculated number of DFDs for the first and second HAMD-17 scores, dividing by two, and multiplying by the number of days between assessments. The QALYs gained were then estimated assuming a gain of 0.41 of a quality-adjusted day for each whole DFD gained. One model [40] transformed DFDs into qualityadjusted days using utility weights from Lave and colleagues [68].

\section{Cost-effectiveness results and uncertainty}

The findings of the reviewed models are presented in Table 1 . The results for the most studied interventions appear to be fairly consistent. Escitalopram was dominant over sertraline (in 2 of 3 analyses [8,39]), duloxetine (in 1 analysis [9]), citalopram (in 9 of 9 analyses $[17,19,20,23,29,38,39,43,44])$, fluoxetine (in 4 of 4 analyses [19,20,25,39]), fluvoxamine (in 1 analysis [45]), generic and controlled-release paroxetine (in 3 of 3 comparisons [31,39]), and venlafaxine extended release (ER) and instant release (IR) (in 7 of 12 comparisons $[19,20,25,29,39,45])$. Escitalopram was cost-effective compared with sertraline [31], SSRIs [31], and venlafaxine $[17,26]$. Escitalopram had similar cost-effectiveness to venlafaxine in two comparisons $[38,43]$ and was less cost-effective in one [31]. Apart from escitalopram, venlafaxine was commonly compared with SSRIs and TCAs and was a dominant strategy in the majority of these comparisons. Among the two studies identified comparing individual SNRIs, duloxetine was dominant over venlafaxine in one comparison [11] and venlafaxine was dominant over duloxetine in the other comparison [41].

All but one study [26] explored parameter uncertainty. Univariate sensitivity analyses were performed in all of 
Table 2 Health-state utility values applied in published models in MDD

\begin{tabular}{|c|c|c|}
\hline Author, year & $\begin{array}{l}\text { Utility value by health } \\
\text { state }^{\mathrm{a}}\end{array}$ & Primary source and method of utility estimation \\
\hline $\begin{array}{l}\text { Armstrong et al., } 2008 \text { [9]; Armstrong } \\
\text { et al., } 2007 \text { [8] }\end{array}$ & $\begin{array}{l}\text { Treated depression: } 0.848 \\
\text { Untreated depression: } 0.58\end{array}$ & Sullivan et al. [39]: EQ-5D \\
\hline Aziz et al., 2005 [10] & $\begin{array}{l}\text { Pharmacotherapy: } \\
\text { Depression: } 0.43 \\
\text { Partial depression: } 0.55 \\
\text { Well: } 0.75 \\
\text { ECT: } \\
\text { Depression: } 0.52 \\
\text { Partial depression: } 0.66 \\
\text { Well: } 0.90\end{array}$ & $\begin{array}{l}\text { Multiple sources: } \\
\text { Hatziandreu et al. [57]; Mazumdar et al. [58]; Judd et al. [59]; McDonald et al. } \\
\text { [60], Sackett and Torrence [61] }\end{array}$ \\
\hline Benedict et al., 2010 [11] & $\begin{array}{l}\text { Remitters: } 0.79 \\
\text { Responders: } 0.68 \\
\text { Non-responders: } 0.55 \\
\text { Staying in remission: } 0.86 \\
\end{array}$ & $\begin{array}{l}\text { Multiple sources: } \\
\text { Eli Lilly, HMBU trial }{ }^{c} \text { (data on file): EQ-5D; Revicki and Wood [62]: standard } \\
\text { gamble }^{d}\end{array}$ \\
\hline Dardennes et al., 2000 [16] & $\begin{array}{l}\text { Remission with follow-up } \\
\text { Preventive strategy: } 0.875 \\
\text { Episodic strategy: } 0.895 \\
\text { Remission without follow- } \\
\text { up: } 0.895^{\mathrm{e}} \\
\text { Recurrence first } 2 \text { months: } \\
0.306^{\mathrm{e}} \\
\text { Recurrence months } 3 \text { and } \\
\text { 4: } 0.725^{\mathrm{e}} \\
\text { Recurrence months } 5 \text { and } \\
6: 0.795^{\mathrm{e}}\end{array}$ & Anton and Revicki [63]: standard gamble \\
\hline Nuijten, 2001 [32] & $\begin{array}{l}\text { Depression, on treatment } \\
\text { SSRI: } 0.70, \text { TCA: } 0.64 \\
\text { In remission, treatment } \\
\text { prolongation } \\
\text { SSRI: } 0.80, \text { TCA: } 0.72 \\
\text { In remission, off treatment: } \\
0.86 \\
\text { Severe depression: } 0.30\end{array}$ & Revicki and Wood [62]: standard gamble ${ }^{d}$ \\
\hline Perlis et al., 2009 [33] & $\begin{array}{l}\text { Recovered } \\
\text { Not on treatment: } 0.88 \\
\text { Disutility of treatment: } 0.04 \\
\text { Depressed } \\
\text { Not on treatment: } 0.63 \\
\text { Disutility on treatment: } \\
0.04\end{array}$ & $\begin{array}{l}\text { Multiple sources: } \\
\text { Bennett et al. [64]; Revicki et al. [65]; Revicki and Wood [62]: standard gamble } \\
\text { Schaffer et al. [66] }\end{array}$ \\
\hline Sado et al., 2009 [35] & $\begin{array}{l}\text { Severe depression: } 0.30^{9} \\
\text { Moderate depression: } 0.63^{9} \\
\text { Response on treatment: } \\
0.80^{\mathrm{g}} \\
\text { Response, no treatment: } \\
0.86^{\mathrm{g}}\end{array}$ & Revicki and Wood [62]: standard gamble ${ }^{d}$ \\
\hline Simon et al., 2006 [36] & $\begin{array}{l}\text { Severe depression: } 0.30 \\
\text { Moderate depression: } 0.63 \\
\text { Remission, treatment: } 0.80 \\
\text { Remission, no treatment: } \\
0.86\end{array}$ & Revicki and Wood [62]: standard gamble \\
\hline Sobocki et al., 2008 [37] & $\begin{array}{l}\text { Well: } 0.86 \\
\text { Episode: } 0.57 \\
\text { Remission: } 0.81\end{array}$ & $\begin{array}{l}\text { Sobocki et al. [67]: } \\
\text { The study administered EQ-5D questionnaire to } 447 \text { patients treated with } \\
\text { antidepressant in primary care. }\end{array}$ \\
\hline Sullivan et al., 2004 [39] & $\begin{array}{l}\text { Treated depression: } 0.848 \\
\text { Untreated depression } \\
\text { decrement: } \\
-0.268 \\
\text { Decrements for ADRs were } \\
\text { applied }\end{array}$ & Sullivan et al. [39]: EQ-5D \\
\hline
\end{tabular}

$\mathrm{ADR}=$ adverse drug reaction; $\mathrm{DFD}=$ disease-free day; $\mathrm{ECT}=$ electroconvulsive therapy; $\mathrm{EQ}-5 \mathrm{D}=$ the EuroQol Five Dimension instrument; $\mathrm{Gl}=$ gastrointestinal; $\mathrm{MDD}=$ major depressive disorder; $\mathrm{MEPS}=$ Medical Expenditure Panel Survey; $\mathrm{QAD}=$ quality-adjusted day; SG = standard gamble, SSRI = selective serotonin reuptake inhibitor; TCA = tricyclic antidepressant; UK = United Kingdom. 


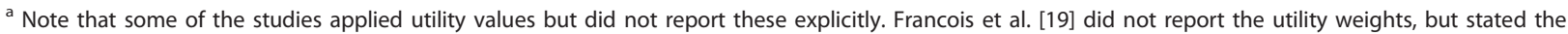
source for these as Quality of Life Perspective Study, Lundbeck (data on file). Lenox-Smith et al. [28] transformed DFDs into utility weights using the methodology from Lave et al. [68] using the following assumptions: a non-depressed subject was assumed to have utility score of 1.0 (perfect health); a subject with major depression was assumed to have a utility score of 0.59 (estimated from literature); subjects were assumed to gain 0.41 of QAD for each depressionfree day. Trivedi et al. [40] transformed DFDs into utility weights using the methodology from Lave et al. [68], assuming a gain of 0.2 to 0.41 of QAD for each DFD.

${ }^{\mathrm{b}}$ Utility values for each health state were derived from a direct analysis of the data in the 2000 MEPS, which provided individual and variance adjustment weights. The EQ-5D was administered via self-administered questionnaire in MEPS.

' Utility study based on trials (Eli Lilly, HMBU trial, data on file) derived utility values for remitters, responders, and non-responders from EQ-5D scores of European patients using the UK tariffs.

${ }^{d}$ Utility of remitters staying in remission was obtained from Revicki and Wood [62]. This study used SG method to generate utilities for 11 hypothetical health states. Health states were varied by severity and medication; 70 patients with MDD or dysthymia who completed $\geq 8$ weeks of antidepressant treatment were recruited.

e The same values for both episodic strategy and preventative strategy.

${ }^{f}$ The SG method was used to generate utilities for hypothetical depression-related health states; 70 MDD patients were interviewed cross-sectionally to provide utilities for these health states.

${ }^{9}$ Both values for combination therapy and antidepressant therapy were assumed to be the same.

h Gl: -0.065; Diarrhoea: -0.044; Dyspepsia: -0.086; Nausea: -0.065; Constipation: -0.065; Sexual: -0.049; Excitation: -0.129; Insomnia: -0.129; Anxiety: -0.129; Drowsiness: -0.085 ; Headache: -0.115 ; Other (average of all ADRs): -0.085 .
}

these studies. Probabilistic sensitivity analyses were performed in approximately three-quarters of studies. Costeffectiveness acceptability curves are used in economic analyses to incorporate the joint uncertainty about the effects and costs [70]; these were presented in 5 of 37 studies [8,11,35-37]. Of the 36 studies, 26 conducted comprehensive sensitivity analyses incorporating all important variables. In 14 of these 26 studies, results were not substantially altered in the sensitivity analyses. In 11 studies, varying clinical input parameters impacted the results while in 7 studies, varying resource use or cost parameters changed the results. Of the 26 studies with the comprehensive sensitivity analyses, eight were the cost-utility studies, of which only two showed sensitivity to changes in utility weights.

\section{Discussion}

We reported the main methodological elements of the published decision-analytic models in MDD. The majority of the reviewed models used a decision-tree structure, largely because the analyses explored the acute and continuation phases of depression and relied on clinical inputs from trials of 6 to 12 weeks in duration. Decision-tree models are appropriate for economic modelling in acute illnesses; however, Markov models are the first choice for pharmacoeconomic analysis of chronic diseases like depression [32]. Markov models provide the advantage over decision-tree models by being able to incorporate longer time horizons, which might be more appropriate given the recurrent nature of depression. The fact that the decision-tree structure is commonly preferred over the Markov structure can be explained by the existing data gaps in the clinical evidence necessary to populate a longer horizon multi-state model. A model design combining both the decisiontree and Markov structures could be utilised to accurately capture the short-term trial data for the acute phase and a longer-term events in the maintenance phase.

Only 18 of 37 studies were conducted from the societal perspective. Both utilisation of health care services and productivity losses are high within the MDD patient population, therefore, it is important to consider a broad cost perspective that captures all of the relevant costs to society. Evidence suggests that employment status is more rapidly affected by depression compared with its effect on utilisation of health care services $[71,72]$. The review of the models suggests that indirect costs have a substantial impact on the outcome of the analysis. In calculation of indirect costs, most models included productivity loss due to absenteeism from work. Productivity loss due to presenteeism (i.e., loss due to patients suffering from symptoms of depression at work resulting in reduced productivity) is also profound $[73,74]$. Costs associated with lost productivity while at work were examined by one model in the sensitivity analysis [37].

The review identified 14 economic analyses that included QALY as a main outcome. The purpose of QALYs is to provide a value- or preference-based outcome measure incorporating trade-offs between quality of life and quantity of life in a common metric [75]. In recent decades, QALY has become the dominant measure of health value in health technology assessment [76]. Current evidence on the health-related quality of life utilised by the identified models appeared to be scarce, particularly for partial response.

There is a need for further cost-effectiveness studies in patients with MDD who have had partial or no response to the first-line therapy. This review revealed a lack of clinical data in inadequate responders to inform such economic models. More studies focusing on evaluating adjunctive MDD therapies would be welcome. The lack of long-term data describing costs and outcomes 
substantially limited the reliability of longer-term MDD models.

The review identified some variability in the methods used by the current models, which inevitably makes the interpretation of results more difficult. This variability was seen around a number of methodological domains. First, studies applied different modelling approaches, including both decision-tree and Markov structures. Second, studies utilised different outcomes, with only 14 studies reporting incremental cost-per-QALY estimates. Third, resource use and cost components included in the models varied substantially between the studies, particularly around the resource use assumptions following failure of the initial therapy. This partially reflects differences in health systems; however, also contributing to this is the absence of a large-scale resource utilisation study in MDD in any of the countries covered by the existing analyses. Finally, primary efficacy data used in the models were derived from single trials, pooled analyses, indirect comparisons, meta-analyses, or combinations of these. The variation in methodology for deriving clinical inputs could be explained by the lack of data for some comparators as well as by the difficulty in performing indirect comparisons due to differences in outcome measures between the trials.

Despite some variability in the methods, the results reported by the identified models were broadly consistent. For venlafaxine, a conflicting result was found in two comparisons with duloxetine, with one study reporting venlafaxine ER as a dominant strategy [41] and one study reporting duloxetine as a dominant strategy compared with venlafaxine ER [11]. Given that all other studies reported fairly consistent results for venlafaxine comparisons, this variation could be due to the differences in patient populations between the two studies, with the former study evaluating first-line interventions and the latter study evaluating patients who failed on first-line SSRI. Similarly, another substantial inconsistency was observed in one of 12 comparisons of escitalopram and venlafaxine [31]. Again, this study examined second-line interventions, suggesting that the cost-effectiveness of interventions evaluated as first-line therapies varies substantially compared with the costeffectiveness of these interventions if used as secondline treatments.

Although model structures varied, overall conclusions regarding the relative cost-effectiveness of interventions were largely consistent. A few exceptions to this were noted. In one study [28], venlafaxine dominated fluoxetine; however, in another study [39], venlafaxine ER was not cost-effective and venlafaxine IR was dominated by fluoxetine. Both models had decision-tree structures, with one [28] using the structure presented by Casciano [15] and the other [39] modelling the initial treatment outcomes in stage one and the treatment of adverse drug reactions in stage two. However, the inconsistency in results also could be due to the use of different measures of response. In comparisons of venlafaxine with SSRIs, one study reported venlafaxine to be dominant [27] while another found venlafaxine to be cost-effective [24]. Both models had decision-tree structures. The longer time horizon in the first study (6 months [27] as opposed to 16 weeks [24]) may have contributed to this difference. Given other parameter differences between these models, it is difficult to conclude with certainty whether or not structural uncertainty is an issue.

The results of the economic models were most sensitive to clinical outcomes than to costs or to utility weights. The uncertainty in key model variables was examined through the use of one-way sensitivity analysis. In approximately half of the models that conducted comprehensive sensitivity analyses, the results were confirmed to be robust. Even if interpreted correctly, however, one-way sensitivity analysis will commonly (in the absence of correlation) underestimate uncertainty, making it particularly vulnerable to false claims that results are robust [77].

Despite the fact that the study by Sullivan and colleagues [39] found that drug-related adverse events have a significant impact on the direct cost and cost-effectiveness of treatments, only three studies considered differences in adverse-event profiles of the individual agents evaluated. Most of the studies modelled discontinuations due to adverse events, as this is equivalent to treatment failure.

Our review had several limitations. Firstly, it did not appraise the quality of the included studies but focused on reporting the methods and data sources used in the models. The second limitation is that we restricted our review to the published literature, therefore excluding searches of the grey literature. Nonetheless, we believe our work provides a comprehensive review of economic models in MDD and could serve as a useful reference for researchers.

\section{Conclusions}

Our review indicated that over the last 10 years a considerable number of economic models have been developed to evaluate the cost and benefits of the interventions for the treatment of MDD. The identified models varied somewhat in their methodology, but the results seemed broadly consistent. In terms of the model input data, the review identified several data gaps, including utility in partial responders, efficacy of second-line treatments, and utilisation estimates obtained from high-quality sources (for example, from observational studies). The review highlighted the difficulty in performing indirect comparisons due to 
differences in outcome measures between the MDD trials. Achieving consistency with this, and consistency in definitions of health states used in MDD clinical trials and utility studies, would be a large step forward.

\section{List of abbreviations used in the text}

CBT: cognitive behavioural therapy; DFD: disease-free day; ECT: electroconvulsive therapy; ER: extended release; HAMD: Hamilton Rating Scale for Depression; IR: instant release; MADRS: Montgomery Asberg Depression Rating Scale; MAOI: monoamine oxidase inhibitor; MDD: major depressive disorder; MeSH: Medical Subject Heading; MT agonist: agonists of the melatonin receptor; NRI: selective norepinephrine reuptake inhibitor; QALY: quality-adjusted life-year; RIMA: reversible inhibitor of monoamine oxidase A; SNRI: selective serotonin and norepinephrine reuptake inhibitor; SSRI: selective serotonin reuptake inhibitor; TCA: tricyclic antidepressant; UK: United Kingdom; US: United States.

\section{Acknowledgements}

We would like to acknowledge the helpful comments provided by Stephen M. Beard, who reviewed the manuscript, and the input by Adam Irving, who checked the manuscript for data consistency and accuracy.

\section{Author details}

${ }^{1}$ RTI Health Solutions, The Pavilion, Towers Business Park, Wilmslow Road, Didsbury, Manchester, M20 2LS, UK. 'Eli Lilly and Company, Global Health Outcomes, Lilly Corporate Center, Indianapolis, IN 46285 USA.

\section{Authors' contributions}

All authors contributed to the study design and coordination. EAZ performed the literature review and led development of the manuscript. SEW contributed to manuscript development. PMC and JB provided an overall direction and critically reviewed the manuscript. All authors have read and approved the final manuscript.

\section{Competing interests}

This study was funded by Eli Lilly and Company. EAZ and SEW served as paid consultants to Eli Lilly and Company and are employees of RTI Health Solutions. PMC and JB are full-time employees and minor shareholders of Eli Lilly and Company.

Received: 22 December 2010 Accepted: 1 February 2012

Published: 1 February 2012

\section{References}

1. World Health Organization: Global burden of disease 2004 summary tables: prevalence for selected causes, in WHO Regions, estimates for 2004. 2008 [http://www.who.int/healthinfo/global_burden_disease/PREV6\% 202004.xls].

2. Hays RD, Wells KB, Sherbourne CD, Rogers W, Spritzer K: Functioning and well-being outcomes of patients with depression compared with chronic general medical illnesses. Arch Gen Psychiatry 1995, 52(1):11-19.

3. Birnbaum HG, Kessler RC, Kelley D, Ben-Hamadi R, Joish VN, Greenberg PE: Employer burden of mild, moderate, and severe major depressive disorder: mental health services utilization and costs, and work performance. Depress Anxiety 2010, 27(1):78-89.

4. Murray CJ, Lopez AD: Evidence-based health policy-lessons from the Global Burden of Disease Study. Science 1996, 274(5288):740-743.

5. National Institute for Health and Clinical Excellence: Depression: the treatment and management of depression in adults. NICE clinical guideline 902009 [http://www.nice.org.uk/nicemedia/live/12329/45888/ 45888.pdf].
6. Bauer M, Bschor T, Pfennig A, Whybrow PC, Angst J, Versiani M, Möller HJ, WFSBP Task Force on Unipolar Depressive Disorders: World Federation of Societies of Biological Psychiatry (WFSBP) guidelines for biological treatment of unipolar depressive disorders in primary care. World J Biol Psychiatry 2007, 8(2):67-104.

7. Barrett B, Byford S, Knapp M: Evidence of cost-effective treatments for depression: a systematic review. J Affect Disord 2005, 84(1):1-13.

8. Armstrong EP, Skrepnek GH, Haim Erder M: Cost-utility comparison of escitalopram and sertraline in the treatment of major depressive disorder. Curr Med Res Opin 2007, 23(2):251-258.

9. Armstrong EP, Malone DC, Erder MH: A Markov cost-utility analysis of escitalopram and duloxetine for the treatment of major depressive disorder. Curr Med Res Opin 2008, 24:1115-1121.

10. Aziz M, Mehringer AM, Mozurkewich E, Razik GN: Cost-utility of 2 maintenance treatments for older adults with depression who responded to a course of electroconvulsive therapy: results from a decision analytic model. Can J Psychiatry 2005, 50(7):389-397.

11. Benedict A, Arellano J, De Cock E, Baird J: Economic evaluation of duloxetine versus serotonin selective reuptake inhibitors and venlafaxine XR in treating major depressive disorder. J Affect Disord 2010, 120:94-104.

12. Borghi J, Guest JF: Economic impact of using mirtazapine compared to amitriptyline and fluoxetine in the treatment of moderate and severe depression in the UK. Eur Psychiatry 2000, 15(6):378-387.

13. Brown M, Van-Loon J, Guest JF: Cost-effectiveness of mirtazapine relative to fluoxetine in the treatment of moderate and severe depression in France. Eur J Psychiat 2000, 14(1):15-25

14. Casciano J, Doyle J, Arikian S, Casciano R: The health economic impact of antidepressant usage from a payer's perspective: a multinational study. Int J Clin Pract 2001, 55:292-299.

15. Casciano R, Arikian SR, Tarride JE, Casciano J, Doyle JJ: Antidepressant selection for major depressive disorder: the budgetary impact on managed care. Drug Benefit Trends 2000, 12(5):6BH-17BH.

16. Dardennes RM, Lafuma A, Fagnani F, Pribil C, Bisserbe JC, Berdeaux G: Economic assessment of a maintenance treatment strategy in prevention of recurrent depressive disorder. Value Health 2000, 3:40-47.

17. Demyttenaere K, Hemels ME, Hudry J, Annemans L: A cost-effectiveness model of escitalopram, citalopram, and venlafaxine as first-line treatment for major depressive disorder in Belgium. Clin Ther 2005, 27:111-124.

18. Doyle JJ, Casciano J, Arikian S, Tarride JE, Gonzalez MA, Casciano R: A multinational pharmacoeconomic evaluation of acute major depressive disorder (MDD): a comparison of cost-effectiveness between venlafaxine, SSRIs and TCAs. Value Health 2001, 4:16-31.

19. Francois $C$, Sintonen $H$, Toumi M: Introduction of escitalopram, a new SSRI in Finland: comparison of cost-effectiveness between the other SSRIs and SNRI for the treatment of depression and estimation of the budgetary impact. J Med Econ 2002, 5:91-107.

20. François C, Toumi M, Aakhus AM, Hansen K: A pharmacoeconomic evaluation of escitalopram, a new selective serotonin reuptake inhibitor. Eur J Health Econ 2003, 4:12-19.

21. Freeman $\mathrm{H}$, Arikian S, Lenox-Smith $\mathrm{A}$ : Pharmacoeconomic analysis of antidepressants for major depressive disorder in the United Kingdom. Pharmacoeconomics 2000, 18:143-148.

22. Haby MM, Tonge B, Littlefield L, Carter R, Vos T: Cost-effectiveness of cognitive behavioural therapy and selective serotonin reuptake inhibitors for major depression in children and adolescents Australian and New Zealand. J Psychiatry 2004, 38:579-591.

23. Hemels ME, Kasper S, Walter E, Einarson TR: Cost-effectiveness analysis of escitalopram: a new SSRI in the first-line treatment of major depressive disorder in Austria. Curr Med Res Opin 2004, 20:869-878.

24. Howard P, Knight C: A clinical- and cost -effectiveness comparison of venlafaxine and selective serotonin reuptake inhibitors (SSRIs) in the management of patients with major depressive disorder from the perspective of an Austrian sickness fund. J Med Econ 2004, 7:93-106.

25. Kongsakon R, Bunchapattanasakda C: The treatment of major depressive disorders (MDD) in Thailand using escitalopram compared to fluoxetine and venlafaxine: a pharmacoeconomic evaluation. J Med Assoc Thai 2008, 91:1117-1128. 
26. Kulp W, von der Schulenburg JMG, Greiner W: Cost-effectiveness of outpatient treatment in depressive patients with escitalopram in Germany. Eur J Health Econ 2005, 6:317-321.

27. Lenox-Smith A, Conway $P$, Knight C: Cost effectiveness of representatives of three classes of antidepressants used in major depression in the UK. Pharmacoeconomics 2004, 22(5):311-319.

28. Lenox-Smith A, Greenstreet L, Burslem K, Knight C: Cost effectiveness of venlafaxine compared with generic fluoxetine or generic amitriptyline in major depressive disorder in the UK. Clin Drug Investig 2009, 29:173-184.

29. Löthgren M, Hemels M, Francois C, Jönsson B: A cost-effectiveness analysis of escitalopram as first line treatment of depression in Sweden. Primary Care Psychiatry 2004, 9:153-161.

30. Machado M, Iskedjian M, Ruiz IA, Einarson TR: The economic impact of introducing serotonin-noradrenaline reuptake inhibitors into the Brazilian National Drug Formulary: cost-effectiveness and budget-impact analyses. Pharmacoeconomics 2007, 25:979-990.

31. Malone DC: A budget-impact and cost-effectiveness model for secondline treatment of major depression. J Manag Care Pharm 2007, 13:S8-S18.

32. Nuijten MJ: Assessment of clinical guidelines for continuation treatment in major depression. Value Health 2001, 4:281-294.

33. Perlis RH, Patrick A, Smoller JW, Wang PS: When is pharmacogenetic testing for antidepressant response ready for the clinic? A costeffectiveness analysis based on data from the STAR*D study (provisional abstract). Neuropsychopharmacology 2009, 34:2227-2236.

34. Rush AJ, Fava M, Wisniewski SR, Lavori PW, Trivedi MH, Sackeim HA, Thase ME, Nierenberg AA, Quitkin FM, Kashner TM, Kupfer DJ, Rosenbaum JF, Alpert J, Stewart JW, McGrath PJ, Biggs MM, ShoresWilson K, Lebowitz BD, Ritz L, Niederehe G, STAR*D Investigators Group: Sequenced treatment alternatives to relieve depression $\left(S_{T A R}{ }^{*} \mathrm{D}\right)$ : rationale and design. Control Clin Trials 2004, 25(1):119-142.

35. Sado M, Knapp M, Yamauchi K, Fujisawa D, So M, Nakagawa A, Kikuchi T, Ono $Y$ : Cost-effectiveness of combination therapy versus antidepressant therapy for management of depression in Japan. Aust N Z J Psychiatry 2009, 43:539-547.

36. Simon J, Pilling S, Burbeck R, Goldberg D: Treatment options in moderate and severe depression: decision analysis supporting a clinical guideline. B J Psychiatry 2006, 189:494-501.

37. Sobocki $P$, Ekman M, Ovanfors A, Khandker R, Jönsson B: The cost-utility of maintenance treatment with venlafaxine in patients with recurrent major depressive disorder. Int J Clin Pract 2008, 62(4):623-32, E-pub 2008 Feb 15.

38. Sorensen J, Stage KB, Damsbo N, Le LA, Hemels ME: A Danish costeffectiveness model of escitalopram in comparison with citalopram and venlafaxine as first-line treatments for major depressive disorder in primary care. Nord J Psychiatry 2007, 61:100-108.

39. Sullivan PW, Valuck R, Saseen J, MacFall HM: A comparison of the direct costs and cost effectiveness of serotonin reuptake inhibitors and associated adverse drug reactions. CNS Drugs 2004, 18(13):911-932.

40. Trivedi MH, Wan GJ, Mallick R, Chen J, Casciano R, Geissler EC, Panish JM: Cost and effectiveness of venlafaxine extended-release and selective serotonin reuptake inhibitors in the acute phase of outpatient treatment for major depressive disorder. J Clin Psychopharmacol 2004, 24:497-506.

41. van Baardewijk M, Vis PM, Einarson TR: Cost effectiveness of duloxetine compared with venlafaxine-XR in the treatment of major depressive disorder. Curr Med Res Opin 2005, 21:1271-1279.

42. Vos T, Corry J, Haby MM, Carter R, Andrews G: Cost-effectiveness of cognitive-behavioural therapy and drug interventions for major depression. Aust N Z J Psychiatry 2005, 39:683-692.

43. Wade AG, Toumi I, Hemels M: A probabilistic cost-effectiveness analysis of escitalopram, generic citalopram and venlafaxine as a first-line treatment of major depressive disorder in the UK. Curr Med Res Opin 2005, 21(4):631-641.

44. Wade AG, Toumi I, Hemels ME: A pharmacoeconomic evaluation of escitalopram versus citalopram in the treatment of severe depression in the United Kingdom. Clin Ther 2005, 27:486-496.

45. Xie F, Despiegel N, Danchenko N, Hansen K: Cost effectiveness analysis of escitalopram compared to venlafaxine and fluvoxamine in treatment of major depressive disorder. Int J Psychiatry Clin Pract 2009, 13:59-69.

46. Khan A, Khan SR, Leventhal RM, Brown WA: Symptom reduction and suicide risk among patients treated with placebo in antipsychotic clinical trials: an analysis of the food and drug administration database. Am J Psychiatry 2001, 158(9):1449-1454.

47. National Institute for Health and Clinical Excellence: Updated Guide to the Methods of Technology Appraisal. 2008 [http://www.nice.org.uk/media/ B52/A7/TAMethodsGuideUpdatedJune2008.pdf].

48. Canadian Agency for Drugs and Technologies in Health: Guidelines for the Economic Evaluation of Health Technologies. Ottawa 2006.

49. Bakish D: New standard of depression treatment: remission and full recovery. J Clin Psychiatry 2001, 62(Suppl 26):5-9.

50. Ballenger JC: Clinical guidelines for establishing remission in patients with depression and anxiety. J Clin Psychiatry 1999, 60(Suppl 22):29-34

51. Arroll B, Elley CR, Fishman T, Goodyear-Smith FA, Kenealy T, Blashki G, Kerse N, Macgillivray S: Antidepressants versus placebo for depression in primary care. Cochrane Database Syst Rev 2009, 3:CD007954.

52. Montgomery SA, Asberg M: A new depression scale designed to be sensitive to change. Br J Psychiatry 1979, 134:382-389.

53. Hamilton M: A rating scale for depression. J Neurol Neurosurg Psychiatry 1960, 23:56-62

54. Guy W, Bonato RR: Manual for the ECDEU Assessment Battery 2 Chevy Chase: National Institute of Mental Health; 1970.

55. Simon GE, Khandker RK, Ichikawa L, Operskalski BH: Recovery from depression predicts lower health services costs. J Clin Psychiatry 2006, 67(8):1226-1231

56. Sobocki P, Ekman M, Ågren H, Krakau I, Runeson B, Mårtensson B, Jönsson B: Resource use and costs associated with patients treated for depression in primary care. Eur J Health Econ 2007, 8:67-76.

57. Hatziandreu EJ, Brown RE, Revicki DA, Turner R, Martindale J, Levine S, Siegel JE: Cost utility of maintenance treatment of recurrent depression with sertraline versus episodic treatment with dothiepin. Pharmacoeconomics 1994, 5(3):249-268.

58. Mazumdar S, Reynolds CF, Houck PR, Frank E, Dew MA, Kupfer DJ: Quality of life in elderly patients with recurrent major depression: a factor analysis of the General Life Functioning Scale. Psychiatry Res 1996, 63(23):183-190.

59. Judd LL, Paulus MJ, Schettler PJ, Akiskal HS, Endicott J, Leon AC, Maser JD, Mueller $\mathrm{T}$, Solomon DA, Keller MB: Does incomplete recovery from first lifetime major depressive episode herald a chronic course of illness? Am J Psychiatry 2000, 157(9):1501-4.

60. McDonald WM, Phillips VL, Figiel GS, Marsteller FA, Simpson CD, Bailey MC Cost-effective maintenance treatment of resistant geriatric depression. Psychiatr Ann 1998, 28:47-52.

61. Sackett DL, Torrence GW: The utility of different health states as perceived by the general public. J Chron Dis 1978, 31:697-704.

62. Revicki DA, Wood M: Patient-assigned health state utilities for depression-related outcomes: differences by depression severity and antidepressant medications. J Affect Disord 1998, 48(1):25-36.

63. Anton SF, Revicki DA: The use of decision analysis in the pharmacoeconomic evaluation of an antidepressant: a cost-effectiveness study of nefazodone. Psychopharmacol Bull 1995, 31(2):249-258.

64. Bennett KJ, Torrance GW, Boyle MH, Guscott R, Moran LA: Development and testing of a utility measure for major, unipolar depression (McSad). Qual Life Res 2000, 9(1):109-120.

65. Revicki DA, Brown RE, Palmer W, Bakish D, Rosser WW, Anton SF, Feeny D: Modelling the cost effectiveness of antidepressant treatment in primary care. Pharmacoeconomics 1995, 8(6):524-540.

66. Schaffer A, Levitt AJ, Hershkop SK, Oh P, MacDonald C, Lanctot K: Utility scores of symptom profiles in major depression. Psychiatry Res 2002 110(2):189-197.

67. Sobocki P, Ekman M, Agren H, Krakau I, Runeson B, Mårtensson B, Jönsson B: Health-related quality of life measured with EQ-5D in patients treated for depression in primary care. Value Health 2007, 10(2):153-160.

68. Lave JR, Frank RG, Schulberg HC, Kamlet MS: Cost-effectiveness of treatments for depression in primary care practice. Arch Gen Psychiatry 1998, 55(7):645-651.

69. Brooks R, EuroQol Group: EuroQol: the current state of play. Health Policy 1996, 37:53-72.

70. Fenwick E, Marshall DA, Levy AR, Nichol G: Using and interpreting costeffectiveness acceptability curves: an example using data from a trial of management strategies for atrial fibrillation. BMC Health Serv Res 2006, 6:52. 
71. Thomas CM, Morris S: Cost of depression among adults in England in 2000. Br J Psychiatry 2003, 183:514-519.

72. Simon GE, Revicki D, Heiligenstein J, Grothaus L, VonKorff M, Katon WJ, Hylan TR: Recovery from depression, work productivity, and health care costs among primary care patients. Gen Hosp Psychiatry 2000, 22(3):153-162.

73. Stewart WF, Ricci JA, Chee E, Hahn SR, Morganstein D: Cost of lost productive work time among US workers with depression. JAMA 2003, 289:3135-344.

74. Kessler RC, Greenberg PE, Mickelson KD, Meneades LM, Wang PS: The effects of chronic medical conditions on work loss and work cutback. J Occup Environ Med 2001, 43:218-225.

75. Weinstein MC, Stason WB: Foundations of cost-effectiveness analysis for health and medical practices. N Engl J Med 1977, 296(13):716-721.

76. Hauber AB: Healthy-years equivalent: wounded but not yet dead. Expert Rev Pharmacoecon Outcomes Res 2009, 9(3):265-269.

77. Claxton K: Exploring uncertainty in cost-effectiveness analysis. Pharmacoeconomics 2008, 26(9):781-798.

doi:10.1186/1478-7547-10-1

Cite this article as: Zimovetz et al:: Methodologies used in costeffectiveness models for evaluating treatments in major depressive disorder: a systematic review. Cost Effectiveness and Resource Allocation 2012 10:1.

\section{Submit your next manuscript to BioMed Central} and take full advantage of:

- Convenient online submission

- Thorough peer review

- No space constraints or color figure charges

- Immediate publication on acceptance

- Inclusion in PubMed, CAS, Scopus and Google Scholar

- Research which is freely available for redistribution

Submit your manuscript at www.biomedcentral.com/submit 